\title{
FUNCTIONAL EQUATIONS FOR ZETA FUNCTIONS OF GROUPS AND RINGS
}

\author{
CHRISTOPHER VOLL
}

\begin{abstract}
We introduce a new method to compute explicit formulae for various zeta functions associated to groups and rings. The specific form of these formulae enables us to deduce local functional equations. More precisely, we prove local functional equations for the subring zeta functions associated to rings, the subgroup, conjugacy and representation zeta functions of finitely generated, torsion-free nilpotent (or $\mathcal{T}$-)groups, and the normal zeta functions of $\mathcal{T}$-groups of class 2 . In particular we solve the two problems posed in [9, Section 5]. We deduce our theorems from a 'blueprint result' on certain $p$-adic integrals which generalises work of Denef and others on Igusa's local zeta function. The Malcev correspondence and a Kirillov-type theory developed by Howe are used to 'linearise' the problems of counting subgroups and representations in $\mathcal{T}$-groups, respectively.
\end{abstract}

\section{INTRODUCTION}

Zeta functions of groups were introduced by Grunewald, Segal and Smith in the 1980s as a tool to study the subgroup growth of finitely generated groups. In [14] the zeta function of a finitely generated group $G$ was defined as the Dirichlet series

$$
\zeta_{G}(s)=\sum_{H \leq G}|G: H|^{-s}
$$

where $s$ is a complex variable and the sum ranges over the finite index subgroups of $G$. Grunewald, Segal and Smith derived results on the zeta functions of finitely generated, torsion-free nilpotent (or $\mathcal{T}$-)groups $G$, and went on to consider variants of (1). These include a $\mathcal{T}$-group's normal zeta function counting only normal subgroups of finite index, and the conjugacy zeta function counting subgroups up to conjugacy. They also developed an analogous theory for rings. (In the current paper, by a ring we mean a finitely generated abelian group with a bi-additive product.) The ideal zeta function of a ring, for instance, generalises the classical Dedekind zeta function of a number field. Much of the subsequent developments in the theory of zeta

Date: February 8, 2008.

2000 Mathematics Subject Classification. 11M41, 20E07, 11S40.

Key words and phrases. Subgroup growth, representation growth, nilpotent groups, Igusa's local zeta function, $p$-adic integration, local functional equations, Kirillov theory. 
functions of groups and rings is documented in the monograph [24] and in the report [9].

Only comparatively recently, Hrushovski and Martin ([17]) started to investigate representation zeta functions of $\mathcal{T}$-groups, enumerating (twist-isoclasses of) finite-dimensional complex characters.

All the zeta functions mentioned so far have the property that they satisfy an Euler product decomposition into local factors, indexed by the primes. For example, for a $\mathcal{T}$-group $G$ we have

$$
\zeta_{G}(s)=\prod_{p \text { prime }} \zeta_{G, p}(s)
$$

where $\zeta_{G, p}(s)=\sum_{H \leq_{p} G}|G: H|^{-s}$ enumerates finite $p$-power index subgroups. All these local zeta functions are known to be rational functions in the parameter $p^{-s}$ with integer coefficients ([14], [17]). In many cases they exhibit a remarkable symmetry: For instance, it had been observed (cf., e.g., [9]) that the local factors of all zeta functions of $\mathcal{T}$-groups $G$ for which explicit formulae are known satisfy a local functional equation of the form

$$
\left.\zeta_{G, p}(s)\right|_{p \rightarrow p^{-1}}=(-1)^{a} p^{b-c s} \zeta_{G, p}(s)
$$

for almost all primes $p$ and suitable integers $a, b, c$ depending only on the Hirsch length of $G$. Here $p \rightarrow p^{-1}$ denotes a formal inversion of the local parameter $p$, which we shall now explain.

The prime example is the case of $G=\mathbb{Z}^{n}$. It is known from [14, Proposition 1.1] that

$$
\zeta_{G}(s)=\prod_{i=0}^{n-1} \zeta(s-i),
$$

where $\zeta(s)=\prod_{p \text { prime }} \frac{1}{1-p^{-s}}$ is the Riemann zeta function. The functional equation

$$
\prod_{i=0}^{n-1} \frac{1}{1-p^{-(i-s)}}=(-1)^{n} p^{\left(\begin{array}{c}
n \\
2
\end{array}\right)-n s} \prod_{i=0}^{n-1} \frac{1}{1-p^{i-s}}
$$

of the local factor at the prime $p$ is easily seen to hold for all primes. Here, as well as in all other cases in which explicit formulae are known to date, the local zeta functions $\zeta_{G, p}(s)$ are in fact rational functions in $p^{-s}$ and $p$, and the left hand side of (2) denotes the rational function obtained by formally inverting both of these two parameters. This 'uniformity' in the prime $p$, however, is not typical: Results of du Sautoy and Grunewald ([8]) show that the dependence of the local (normal) zeta functions of $\mathcal{T}$-groups on the primes will, in general, reflect the variation of the number of $\mathbb{F}_{p}$-points of certain algebraic varieties defined over $\mathbb{F}_{p}$, which may be far from polynomial in the prime $p$ (see also [6] and [7]). In [31] we produced examples of normal zeta functions of $\mathcal{T}$-groups of nilpotency class 2 (or $\mathcal{T}_{2}$-groups) which exhibit functional equations similar to (2) and which are not uniform. Indeed, the local factors of these zeta functions are rational functions in $p^{-s}$, whose coefficients involve the numbers $b_{V}(p)$ of $\mathbb{F}_{p}$-rational points of certain smooth projective varieties $V$ over $\mathbb{F}_{p}$ 
which are, in general, not polynomials in $p$. By the Weil conjectures, these numbers may be expressed as alternating sums of Frobenius eigenvalues. The operation $p \rightarrow p^{-1}$ is performed by inverting these eigenvalues. In the special ('uniform') case that the $b_{V}(p)$ are in fact polynomials in $p$, this specialises to an inversion of the prime $p$.

Only a single example of a representation zeta function of a $\mathcal{T}$-group seems to have appeared in print so far: In [17] Hrushovski and Martin derive a formula for the representation zeta function of the discrete Heisenberg group in terms of the Riemann zeta function and its inverse (cf. Example 1.2).

According to du Sautoy and Segal, to find an explanation for the phenomenon of local functional equations for zeta functions of groups and rings is "one of the most intriguing open problems in this area" ([11, p. 274]). In the current paper we prove that local functional equations hold for (almost all factors of) the following zeta functions:

(A) zeta functions of rings (and, as a corollary, of $\mathcal{T}$-groups),

(B) conjugacy zeta functions of $\mathcal{T}$-groups,

(C) normal zeta functions of $\mathcal{T}_{2}$-groups and

(D) representation zeta functions of $\mathcal{T}$-groups.

By proving (A) and (C) we solve Problems 5.1 and 5.2 posed in [9]. In its given generality, $(\mathrm{C})$ is best possible: It is known that the normal zeta functions of nilpotent groups of class 3 may or may not satisfy local functional equations (cf. [13]). To determine the exact scope of this intriguing symmetry for ideal zeta functions of rings remains a challenging open problem.

We achieve our results by showing that all of the above-mentioned zeta functions may be expressed in terms of certain $p$-adic integrals, generalising Igusa's local zeta function. Given a non-constant polynomial $f(\mathbf{y}) \in$ $\mathbb{Z}\left[y_{1}, \ldots, y_{m}\right]$, its associated Igusa local zeta function is the $p$-adic integral

$$
\int_{\mathbb{Z}_{p}^{m}}|f(\mathbf{y})|^{s}|\mathrm{~d} \mathbf{y}|,
$$

where $s$ is a complex variable, || stands for the $p$-adic absolute value and $|\mathrm{dy}|$ denotes the (additive) Haar measure on $\mathbb{Z}_{p}^{m}$, the affine $m$-space over the $p$-adic integers $\mathbb{Z}_{p}$. This $p$-adic integral is closely related to the Poincaré series counting $p$-adic points on the hypersurface defined by $f$ (cf. [4]). We prove functional equations for these integrals by generalising results by Denef and others on Igusa's local zeta function.

The integrals considered in the present paper are quite different from the 'cone integrals' introduced by du Sautoy and Grunewald in [8] (see Section 1.2 for further details).

1.1. Detailed statement of results. Let $L$ be a ring. Its (subring) zeta function is defined to be the Dirichlet series

$$
\zeta_{L}(s)=\sum_{H \leq L}|L: H|^{-s}
$$


where the sum ranges over all subrings $H$ of finite index in $L$, and $s$ is a complex variable. This zeta function decomposes naturally as an Euler product, indexed by the primes:

$$
\zeta_{L}(s)=\prod_{p \text { prime }} \zeta_{L, p}(s)
$$

where $\zeta_{L, p}(s)=\zeta_{L \otimes \mathbb{Z}_{p}}(s)$. Grunewald, Segal and Smith proved in [14] that each local factor is a rational function in $p^{-s}$ with integral coefficients. Our first main Theorem is

Theorem A. Let $L$ be a ring of torsion-free rank $n$. Then there are smooth projective varieties $V_{t}, t \in\{1, \ldots, m\}$, defined over $\mathbb{Q}$, and rational functions $W_{t}(X, Y) \in \mathbb{Q}(X, Y)$ such that for almost all primes $p$ the following hold.

(a) Denoting by $b_{t}(p)$ the number of $\mathbb{F}_{p}$-rational points of $\overline{V_{t}}$, the reduction $\bmod p$ of $V_{t}$, we have

$$
\zeta_{L, p}(s)=\sum_{t=1}^{m} b_{t}(p) W_{t}\left(p, p^{-s}\right) .
$$

(b) Setting $b_{t}\left(p^{-1}\right):=p^{-\operatorname{dim}\left(V_{t}\right)} b_{t}(p)$ the following functional equation holds:

$$
\left.\zeta_{L, p}(s)\right|_{p \rightarrow p^{-1}}=(-1)^{n} p^{\left(\begin{array}{c}
n \\
2
\end{array}\right)-n s} \zeta_{L, p}(s) .
$$

The novelty of this result is that it allows us to deduce the equations (4). In [8], du Sautoy and Grunewald gave formulae akin to (3) for, inter alia, the local factors of $\zeta_{L}(s)$. Their proof depends on a representation of local zeta functions through certain $p$-adic integrals called 'cone integrals' which in general will not satisfy functional equations like (4). See [13, Chapter 4] for a discussion of functional equations for cone integrals.

Given a ring $L$ we cannot, in general, pin down the primes $p$ which have to be excluded in Theorem A. On the other hand, any prime $p$ will be among the primes for which Theorem A, applied to the ring $p L$, makes no assertion.

We also note that the definition in part (b) of Theorem A is consistent with our above explanation of the operation $p \rightarrow p^{-1}$. Indeed, by the Weil conjectures, the numbers $b_{t}(p)$ may be expressed as alternating sums of Frobenius eigenvalues. These complex numbers satisfy certain symmetries which are reflected by the functional equations for the Weil zeta functions of the varieties $\overline{V_{t}}$. Had the expressions $b_{t}\left(p^{-1}\right)$ been defined as the numbers obtained from inverting these eigenvalues, the identities $b_{t}\left(p^{-1}\right)=p^{-\operatorname{dim}\left(V_{t}\right)} b_{t}(p)$ would follow from these symmetries (see the remarks preceding Theorem 2.3 for details).

Example 1.1. Theorem A applies in particular to the zeta functions of 'simple' Lie algebras over $\mathbb{Z}$ such as $\mathfrak{s l}(d, \mathbb{Z})$. The only such Lie algebra for which a functional equation as in (4) had been previously established is $L=\mathfrak{s t}(2, \mathbb{Z})$ 
(cf. $\left.[12]^{1}\right)$ :

$$
\zeta_{L}(s)=\zeta(s) \zeta(s-1) \zeta(2 s-1) \zeta(2 s-2)\left(1+3 \cdot 2^{1-2 s}-2^{3-3 s}\right) \prod_{p \neq 2}\left(1-p^{1-3 s}\right) .
$$

In fact, $\mathfrak{s l}(2, \mathbb{Z})$ seems to be the only non-soluble Lie ring whose subring zeta function has been computed explicitly. Note that the functional equation fails for $p=2$. A similar phenomenon may occur if one studies the zeta function of a $\mathbb{Z}_{p^{-}}$-algebra: In [21] Klopsch computes the zeta function of a maximal $\mathbb{Z}_{p^{-}}$ order in a central simple $\mathbb{Q}_{p}$-division algebra of index 2 . The fact that this zeta function does not satisfy a functional equation of the form (4) reflects the fact that it is not the 'generic' local factor of the zeta function of a ring.

Klopsch and the present author have unified and generalised the above examples. In [22] they gave a formula for the zeta function of an arbitrary 3 -dimensional $\mathbb{Z}_{p}$-Lie algebra, based on the proof of Theorem A.

An important corollary of Theorem A is to the theory of zeta functions of finitely generated, torsion-free nilpotent (or $\mathcal{T}$-) groups. In [14, Theorem 4.1] it was shown that, given a $\mathcal{T}$-group $G$ of Hirsch length $n$, there is a Lie subring $L=L(G)$, lying as a full $\mathbb{Z}$-lattice in an $n$-dimensional Lie algebra $\mathcal{L}(G)$ over $\mathbb{Q}$ such that for almost all primes $p$

$$
\zeta_{G, p}(s)=\zeta_{L, p}(s) \text {. }
$$

Thus we obtain

Corollary 1.1. Let $G$ be a $\mathcal{T}$-group of Hirsch length $n$. For all but finitely many primes $p$

$$
\left.\zeta_{G, p}(s)\right|_{p \rightarrow p^{-1}}=(-1)^{n} p^{\left(\begin{array}{c}
n \\
2
\end{array}\right)-n s} \zeta_{G, p}(s) .
$$

Theorem A is itself an instance of an application of a 'reciprocity' result (Corollary 2.3 to Theorem 2.3) establishing certain functional equations for a family of $p$-adic integrals. Theorem 2.3 may be viewed as a generalisation of Stanley's 'reciprocity theorem for linear homogeneous diophantine equations' ([28, Theorem 4.6.14]), which we now briefly explain. Given a set of simultaneous linear homogeneous diophantine equations in $n$ indeterminates, say, one may encode their non-negative (positive) solutions in a rational generating function $E(\mathbf{x})\left(\bar{E}(\mathbf{x})\right.$, respectively), where $\mathbf{x}=\left(x_{1}, \ldots, x_{n}\right)$ is a vector of formal variables. More precisely, one defines

$$
E(\mathbf{x}):=\sum_{\alpha \in \mathbb{N}_{0}^{n} \cap \mathscr{C}} \mathbf{x}^{\alpha} \text { and } \bar{E}(\mathbf{x}):=\sum_{\alpha \in \mathbb{N}^{n} \cap \mathscr{C}} \mathbf{x}^{\alpha},
$$

where $\alpha=\left(\alpha_{1}, \ldots, \alpha_{n}\right), \mathbf{x}^{\alpha}=x_{1}^{\alpha_{1}} \ldots x_{n}^{\alpha_{n}}$ and $\mathscr{C}$ is the cone of all nonnegative real solutions to the given set of equations. Stanley's reciprocity theorem states that, if $\bar{E}(\mathbf{x}) \neq 0$, then

$$
\bar{E}(1 / \mathbf{x})=(-1)^{\operatorname{dim} \mathscr{C}} E(\mathbf{x}) .
$$

\footnotetext{
${ }^{1}$ The denominator of $P\left(2^{-s}\right)$ in $\left[9\right.$, Equation (7)] should read $1-2^{1-3 s}$.
} 
Our generalisation is obtained using (a variant of) Stanley's result and an explicit formula of the form (3) for the integrals in question (Corollary 2.1 of Theorem 2.2). This formula in turn is inspired by and generalises work of Denef ([3]), Denef and Meuser ([5]) and Veys and Zuniga-Galindo ([29]) on Igusa's local zeta function. While Igusa's local zeta functions associated to homogeneous polynomial mappings may be expressed as integrals over projective space, the $p$-adic integrals considered in the current paper reduce to integrals over the complete flag variety $\mathrm{GL}_{n} / B$, where $B$ is a Borel subgroup. In a sense this explains the factor $p^{\left(\begin{array}{c}n \\ 2\end{array}\right)}$ in (4).

A variant of the problem of counting subgroups consists in counting subgroups only up to conjugacy. Let $G$ be a $\mathcal{T}$-group. The conjugacy zeta function of a $\mathcal{T}$-group $G$ is defined as

$$
\zeta_{G}^{\mathrm{cc}}(s)=\sum_{H \leq G}|G: H|^{-s}\left|\mathcal{C}_{G}(H)\right|^{-1}
$$

where $\left|\mathcal{C}_{G}(H)\right|$ is the size of the conjugacy class of $H$. It is known ([14, Remark on p. 189]) that $\zeta_{G}^{\mathrm{cc}}(s)$ also has an Euler product decomposition into local factors $\zeta_{G, p}^{c c}(s)$ which are all rational in $p^{-s}$. By applying the results of Section 2 we shall prove

Theorem B. Let $G$ be a $\mathcal{T}$-group of Hirsch length $n$. For all but finitely many primes $p$

$$
\left.\zeta_{G, p}^{\mathrm{cc}}(s)\right|_{p \rightarrow p^{-1}}=(-1)^{n} p^{\left(\begin{array}{c}
n \\
2
\end{array}\right)-n s} \zeta_{G, p}^{\mathrm{cc}}(s) .
$$

As a third application of our rather technical 'blueprint result' Theorem 2.2 and its applications we deduce functional equations for normal zeta functions of $\mathcal{T}_{2}$-groups. The normal zeta function of a $\mathcal{T}$-group $G$ is defined as

$$
\zeta_{G}^{\triangleleft}(s)=\sum_{H \triangleleft G}|G: H|^{-s}
$$

where the sum ranges over the normal subgroups $H$ of finite index in $G$. It also satisfies an Euler product decomposition. We prove

Theorem C. Let $G$ be a $\mathcal{T}_{2}$-group of Hirsch length $n$ with centre $Z(G)$ such that $G / Z(G)$ has torsion-free rank $d$. For all but finitely many primes $p$

$$
\left.\zeta_{G, p}^{\triangleleft}(s)\right|_{p \rightarrow p^{-1}}=(-1)^{n} p^{\left(\begin{array}{c}
n \\
2
\end{array}\right)-(d+n) s} \zeta_{G, p}^{\triangleleft}(s) .
$$

As mentioned above, a functional equation may or may not hold for normal zeta functions of class greater than three. See [13, Chapter 2] for examples and [13, Theorem 4.44] for a conjectural form in case it does hold.

The fourth and last application in this paper of Theorem 2.2 and its consequences is concerned with representation zeta functions of $\mathcal{T}$-groups, which we shall now explain. Given a $\mathcal{T}$-group $G$, we denote by $R_{n}(G)$ the set of $n$-dimensional complex characters of $G$. Given $\sigma_{1}, \sigma_{2} \in R_{n}(G)$, we say that $\sigma_{1}$ and $\sigma_{2}$ are twist-equivalent if there exists a linear character $\chi \in R_{1}(G)$ 
such that $\sigma_{1}=\chi \sigma_{2}$. The classes of this equivalence relation are called twistisoclasses. We say that a character $\sigma$ of a representation $\rho$ of $G$ factors through a finite quotient of $G$ if $\rho$ factors through it. The set $R_{n}(G)$ has the structure of a quasi-affine complex algebraic variety whose geometry was analysed by Lubotzky and Magid in [23]. They proved

Theorem [23, Theorem 6.6] Let $G$ be a $\mathcal{T}$-group. For every $n \in \mathbb{N}$ there is a finite quotient $G(n)$ of $G$ such that every $n$-dimensional irreducible character of $G$ is twist-equivalent to one that factors through $G(n)$. In particular, the number of twist-isoclasses of irreducible $n$-dimensional characters is finite.

Let us call this number $a_{n}$. The representation zeta function of $G$ is defined (cf. [17]) by

$$
\zeta_{G}^{\mathrm{irr}}(s):=\sum_{n=1}^{\infty} a_{n} n^{-s} .
$$

It follows from the above theorem and [2, (10.33)] that the function $n \mapsto a_{n}$ is multiplicative and thus

$$
\zeta_{G}^{\mathrm{irr}}(s)=\prod_{p \text { prime }} \zeta_{G, p}^{\mathrm{irr}}(s)
$$

where

$$
\zeta_{G, p}^{\mathrm{irr}}(s):=\sum_{n=0}^{\infty} a_{p^{n}} p^{-s n}
$$

Example 1.2. ([17, Example 8.12], [25, Theorem 5]) Let

$$
\left.H=\left\langle x_{1}, x_{2}, y\right|\left[x_{1}, x_{2}\right]=y, \text { all other }[,] \text { trivial }\right\rangle
$$

be the discrete Heisenberg group. Then

$$
\zeta_{H}^{\mathrm{irr}}(s)=\sum_{n=1}^{\infty} \phi(n) n^{-s}=\frac{\zeta(s-1)}{\zeta(s)}=\prod_{p \text { prime }} \frac{1-p^{-s}}{1-p^{1-s}},
$$

where $\phi$ denotes the Euler totient function.

By a model-theoretic result of Hrushovski and Martin [17, Theorem 8.4], the local representation zeta functions of a $\mathcal{T}$-group are known to be rational functions in $p^{-s}$ with integer coefficients. By expressing $\zeta_{G, p}^{\text {irr }}(s)$ in terms of $p$-adic integrals to which Theorem 2.2 is applicable we shall prove

Theorem D. Let $G$ be a $\mathcal{T}$-group with derived group $G^{\prime}=[G, G]$ of Hirsch length $n$. Then for almost all primes $p$

$$
\left.\zeta_{G, p}^{\mathrm{irr}}(s)\right|_{p \rightarrow p^{-1}}=p^{n} \zeta_{G, p}^{\mathrm{irr}}(s) .
$$


1.2. Outline of methodology and related work. We briefly describe how we relate the problems solved in Theorems A, B, C and D to problems about $p$-adic integrals.

To count the subrings of $p$-power index in a ring $L$ of rank $n$, we observe that it is enough to keep track of the index of the largest $\mathbb{Z}_{p}$-subalgebra of $L \otimes \mathbb{Z}_{p}$ in each given homothety class of lattices in the $p$-adic vector space $\mathbb{Q}_{p}^{n}$. Using the action of the group $\Gamma:=\mathrm{GL}_{n}\left(\mathbb{Z}_{p}\right)$ on the set of homothety classes, we show that the latter problem reduces to counting polynomial congruences in finite quotients of $\Gamma$. This counting problem translates into the problem of computing a $p$-adic integral in very much the same fashion as the problem of counting polynomial congruences in affine space translates to the problem of computing Igusa's local zeta function (cf. [4, Section 1.2]). It proved helpful to think of homothety classes of lattices as the vertices of the BruhatTits building of $\mathrm{SL}_{n}\left(\mathbb{Q}_{p}\right)$, and to partition the vertex set into finitely many parts according to their position relative to the 'root class' $\left[L \otimes \mathbb{Z}_{p}\right]$. As we remarked above, this approach differs decisively from the 'cone integrals' introduced by du Sautoy and Grunewald in [8]. Their analysis rests on a basis dependent parametrisation of $p$-power index subrings of a given ring in terms of upper-triangular matrices over the $p$-adic integers satisfying certain divisibility conditions ('cone conditions').

To count subgroups up to conjugacy in a $\mathcal{T}$-group $G$ we use the fact that, for almost all primes $p$,

$$
\zeta_{G, p}^{\mathrm{cc}}(s)=\zeta_{L, p}^{\mathrm{cc}}(s)
$$

where $L=L(G)$ is the Lie ring associated to $G$ (cf. the remark preceding Corollary 1.1), and

$$
\zeta_{L, p}^{\mathrm{cc}}(s)=\sum_{H \leq L \otimes \mathbb{Z}_{p}}\left|L \otimes \mathbb{Z}_{p}: H\right|^{-s}\left|L \otimes \mathbb{Z}_{p}: \mathcal{N}_{L \otimes \mathbb{Z}_{p}}(H)\right|^{-1},
$$

where $H$ ranges over the subalgebras of $L \otimes \mathbb{Z}_{p}$ of finite index and $\mathcal{N}_{L \otimes \mathbb{Z}_{p}}(H)$ is the normaliser of $H$ in $L \otimes \mathbb{Z}_{p}$. We thus have to keep track both of the largest subring of $L \otimes \mathbb{Z}_{p}$ in each given homothety class and of the class' normaliser. The index of the latter is given by the index of a system of linear congruences. Enumerating these indices, in turn, may be achieved by counting the elementary divisors of matrices of linear forms, encoding the group's commutator structure. In Proposition 2.2 we show that, slightly more generally, the generating functions enumerating elementary divisors of matrices of polynomial forms of the same degree may be expressed in terms of $p$-adic integrals associated to degeneracy loci of these matrices, to which Corollary 2.4 is applicable.

In order to count normal subgroups in class-2-nilpotent groups we develop an idea first introduced in [30]. There it was shown that it suffices to evaluate a weight function on the set of homothety classes of lattices in the centre $Z\left(L \otimes \mathbb{Z}_{p}\right)$ of the $\mathbb{Z}_{p}$-Lie algebra $L \otimes \mathbb{Z}_{p}$, where $L$ is the associated Lie ring. The weight associated to a vertex in the appropriate affine Bruhat-Tits building corresponding to a given class is again given by the index of a system of linear congruences. 
As mentioned above, the validity of our main Theorems $\mathrm{A}$ and $\mathrm{C}$ had been known in many special cases. We refer the reader to the numerous examples collected in [13]. In this research monograph du Sautoy and Woodward also present a conjecture on functional equations for cone integrals that would explain functional equations for normal zeta functions of nilpotent of class greater than 2.

The key to Theorem D is to use 'Kirillov-theory' developed by Howe [16] to translate the problem of counting irreducible representations of $G$ to the problem of counting co-adjoint orbits in the dual of the Lie algebra associated to $G$ by the Malcev correspondence. We use the fact - also established by Howe - that the sizes of co-adjoint orbits may be expressed in terms of the indices of the radicals of certain anti-symmetric forms on the Lie algebra. These may also be described in terms of elementary divisors of matrices encoding the structure of the Lie algebra.

Representation zeta functions of nilpotent groups have not been studied until fairly recently, and [17] seems to be the only reference so far on this topic. The idea of using Kirillov-theory to study representation zeta functions of groups, however, has been successfully employed before. Jaikin-Zapirain proved in [19] the rationality of representation zeta functions for certain compact $p$-adic analytic groups using a Kirillov-type correspondence developed by Howe ([15]) for these groups. In [17] Hrushovski and Martin suggest that Jaikin-Zapirain's work may be adapted to prove rationality of local representation zeta functions for $\mathcal{T}$-groups, too.

Among the variants of the zeta function (1) of a $\mathcal{T}$-group $G$ considered in [14] is also the zeta function $\zeta_{G}^{\wedge}(s)$, enumerating subgroups of finite index whose profinite completion is isomorphic to the profinite completion of $G$. In [10] du Sautoy and Lubotzky proved a functional equations for the local factors of $\zeta_{G}^{\wedge}(s)$ for a class of $\mathcal{T}$-groups. Their work is based on a reduction of the problem of computing these zeta functions to the problem of computing certain $p$-adic integrals over the group's algebraic automorphism group, generalising work of Igusa's ([18]). The functional equation for the local factors of these zeta functions arises from a symmetry in the root system of the associated Weyl groups. An argument of this kind (albeit only for the Weyl groups of type $A$ ) is also used in the present paper to deduce Corollary 2.3. We do not know whether the zeta functions $\zeta_{G}^{\wedge}(s)$ (for reasonably large classes of $\mathcal{T}$-groups) may be described by the $p$-adic integrals studied in the current paper. In [1] Berman extends the approach taken in [10], proving uniformity and local functional equations for these zeta functions for a wider class of nilpotent groups than previously considered.

The fact that we have to disregard finitely many primes in most of our results has two reasons: Firstly, our Theorems 2.1 and 2.2 upon which Theorem 2.3 and its corollaries are based are valid only for primes for which a certain principalisation of ideals has good reduction. Secondly, we are forced to ignore finitely many primes in order to transfer between the $\mathcal{T}$-group $G$ and its associated Lie algebra. 
1.3. Layout of the paper and notation. In Section 2 we first develop, in Theorems 2.1 and 2.2, explicit formulae for certain families of $p$-adic integrals generalising Igusa's local zeta function. These two results - which may be understood as close analogues of Theorems 2 and 3 in [5] - form the technical core of the paper. We use them to establish, in Theorem 2.3, an 'inversion property' enjoyed by the $p$-adic integrals considered. In Corollaries 2.3 and 2.4 we exploit this property to deduce functional equations for certain linear combinations of the $p$-adic integrals in question. The remainder of the paper is dedicated to showing how Theorem 2.2 may be used as a template to describe various kinds of zeta functions. In the second part of Section 2 we give a first application of this idea to the problem of counting elementary divisors of matrices of forms (Proposition 2.2). In the four subsections of Section 3 we prove Theorems A, B, C and D, respectively.

We use the following notation.

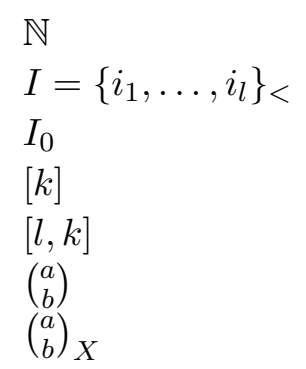

$\left(\begin{array}{l}n \\ I\end{array}\right)_{X}$

$S_{n}$

$M^{t}$

$\mathbb{Z}_{p}$

$\mathbb{Q}_{p}$

$[\Lambda]$

$K$

$R$

$\frac{P}{K}$

K

$\delta_{P}$ the set $\{1,2, \ldots\}$ of natural numbers

the set $I$ of natural numbers $i_{1}<\cdots<i_{l}$

the set $I \cup\{0\}$ for $I \subseteq \mathbb{N}$

the set $\{1, \ldots, k\}, k \in \mathbb{N}$

the set $\{l, \ldots, k\}, k, l \in \mathbb{N}$

the binomial coefficient for $a, b \in \mathbb{N}_{0}$

the polynomial $\prod_{i=0}^{b-1}\left(1-X^{a-i}\right) /\left(1-X^{b-i}\right)$,

where $a, b \in \mathbb{N}_{0}$ with $a \geq b$

Note: The q-binomial coefficient or Gaussian

polynomial $\left(\begin{array}{l}a \\ b\end{array}\right)_{q}$ gives the number of

subspaces of dimension $b$ in $\mathbb{F}_{q}^{a}$.

the polynomial $\left(\begin{array}{l}n \\ i_{l}\end{array}\right)_{X}\left(\begin{array}{c}i_{l} \\ i_{l-1}\end{array}\right)_{X} \ldots\left(\begin{array}{c}i_{2} \\ i_{1}\end{array}\right)_{X}$,

for $n \in \mathbb{N}, I=\left\{i_{1}, \ldots, i_{l}\right\}_{<} \subseteq[n-1]$

Note: $\left(\begin{array}{l}n \\ I\end{array}\right)_{q}$ gives the number of flags of type $I$ in $\mathbb{F}_{q}^{n}$.

the symmetric group on $n$ letters

the transpose of a matrix $M$

the ring of $p$-adic integers ( $p$ a prime)

the field of $p$-adic numbers

the homothety class $\mathbb{Q}_{p}^{*} \Lambda$ of a (full) lattice $\Lambda$ in $\mathbb{Q}_{p}^{n}$

a finite extension of the field $\mathbb{Q}_{p}$

the valuation ring of $K$

the maximal ideal of $R$

the residue field $R / P$, of cardinality $q$

a number field

the 'Kronecker delta' which is equal to 1 if

the property $P$ holds and equal to 0 otherwise.

Given a set $\mathbf{f}$ of polynomials and a polynomial $g$, we write $g \mathbf{f}$ for $\{g f \mid f \in \mathbf{f}\}$, and (f) for the polynomial ideal generated by $\mathbf{f}$. 


\section{Functional EQUATIONS For SOME $p$-ADiC INTEGRALS}

2.1. A blueprint result. In this section we study a family of $p$-adic integrals generalising Igusa's local zeta functions. We first introduce some more notation. Let $p$ be a prime and $K$ be a finite extension of the field $\mathbb{Q}_{p}$ of $p$-adic numbers. Let $R=R_{K}$ denote the valuation ring of $K, P=P_{K}$ the maximal ideal of $R$, and $\bar{K}$ the residue field $R / P$. The cardinality of $\bar{K}$ will be denoted by $q$.

For $x \in K$, let $v(x)=v_{P}(x) \in \mathbb{Z} \cup\{\infty\}$ denote the $P$-adic valuation of $x$, and $|x|:=q^{-v(x)}$. For a finite set $\mathcal{S}$ of elements of $K$, we set $\|\mathcal{S}\|:=$ $\max \{|s| \mid s \in \mathcal{S}\}$. Fix $k, m, n \in \mathbb{N}$. For each $\kappa \in[k]$, let $\left(\mathbf{f}_{\kappa \iota}\right)_{\iota \in I_{\kappa}}$ be a finite family of finite sets of polynomials in $K\left[y_{1}, \ldots, y_{m}\right]$, and let $x_{1}, \ldots, x_{n-1}$ be independent variables. Also, for $i \in[n-1]$ we fix non-negative integers $e_{i \kappa \iota}$. For a set $I=\left\{i_{1}, \ldots, i_{l}\right\}<\subseteq[n-1], \kappa \in[k]$, we set

$$
\mathbf{g}_{\kappa, I}(\mathbf{x}, \mathbf{y})=\bigcup_{\iota \in I_{\kappa}}\left(\prod_{i \in I} x_{i}^{e_{i \kappa \iota}}\right) \mathbf{f}_{\kappa \iota}(\mathbf{y}) \text {. }
$$

Let $W \subseteq R^{m}$ be a subset which is a union of cosets $\bmod P^{m}$ and $\mathbf{s}=$ $\left(s_{1}, \ldots, s_{k}\right)$ be independent complex variables. We then define

$$
Z_{W, K, I}(\mathbf{s}):=\int_{P^{l} \times W} \prod_{\kappa \in[k]}\left\|\mathbf{g}_{\kappa, I}(\mathbf{x}, \mathbf{y})\right\|^{s_{\kappa}}\left|\mathrm{d} \mathbf{x}_{I}\right||\mathrm{d} \mathbf{y}|
$$

where $\left|\mathrm{d} \mathbf{x}_{I}\right|=\left|\mathrm{d} x_{i_{1}} \wedge \cdots \wedge \mathrm{d} x_{i_{l}}\right|$ is the Haar measure on $K^{l}$ normalised so that $R^{l}$ has measure 1 (and thus $P^{l}$ has measure $q^{-l}$ ), and $|\mathrm{d} \mathbf{y}|=\left|\mathrm{d} y_{1} \wedge \cdots \wedge \mathrm{d} y_{m}\right|$ is the (normalised) Haar measure on $K^{m}$. It is well-known that $Z_{W, K, I}(\mathbf{s})$ is a rational function in $q^{-s_{\kappa}}, \kappa \in[k]$, with integral coefficients.

We now assume that the polynomials constituting the sets $\mathbf{f}_{\kappa \iota}$ are in fact defined over a number field $F$. We may consider the local zeta functions $Z_{W, K, I}(\mathbf{s})$ for all non-archimedean completions $K$ of $F$. In the remainder of this section we shall derive formulae for $Z_{W, K, I}(\mathbf{s})$, valid for almost all completions $K$ of $F$ under this and further assumptions. They are essentially based on the formulae Denef gave for Igusa's local zeta function

$$
Z(s)=\int_{R^{m}}|f(\mathbf{y})|^{s}|\mathrm{~d} \mathbf{y}|
$$

in [3, Theorem 3.1], using the concept of resolution of singularities for the hypersurface defined by $f$. In the case where the single polynomial $f$ is replaced by a finite set of polynomials f , Veys and Zuniga-Galindo ([29, Theorem 2.10]) gave an analogous formula, using instead the concept of principalisation of ideals, which we briefly recall.

Theorem [33, Theorem 1.0.1] Let $\mathcal{I}$ be a sheaf of ideals on a smooth algebraic variety $X$. There exists a principalisation $(Y, h)$ of $\mathcal{I}$, that is, a sequence

$$
X=X_{0} \stackrel{h_{1}}{\longleftarrow} X_{1} \longleftarrow \cdots \stackrel{h_{\iota}}{\longleftarrow} X_{\iota} \longleftarrow \cdots \stackrel{h_{r}}{\longleftarrow} X_{r}=Y
$$

of blow-ups $h_{\iota}: X_{\iota} \rightarrow X_{\iota-1}$ of smooth centres $C_{\iota-1} \subset X_{\iota-1}$ such that 
a) The exceptional divisor $E_{\iota}$ of the induced morphism $h^{\iota}=h_{\iota} \circ \cdots \circ h_{1}$ : $X_{\iota} \rightarrow X$ has only simple normal crossings and $C_{\iota}$ has simple normal crossings with $E_{\iota}$.

b) Setting $h=h_{r} \circ \cdots \circ h_{1}$, the total transform $h^{*}(\mathcal{I})$ is the ideal of a simple normal crossing divisor $\widetilde{E}$. If the subscheme determined by $\mathcal{I}$ has no components of codimension one, then $\widetilde{E}$ is an $\mathbb{N}$-linear combination of the irreducible components of the divisor $E_{r}$.

Also recall the definition [3, Definition 2.2 (mutatis mutandis)] of a principalisation $(Y, h)$ with good reduction $\bmod P$ if $\mathcal{I}$ and $(Y, h)$ are defined over a $p$-adic field $K$. Note that, given a principalisation $(Y, h)$ for $\mathcal{I}$ defined over a number field $F,(Y, h)$ will have good reduction $\bmod P$ (where $P$ is the maximal ideal in the ring of integers of the completion of $F$ at $K$ ) for almost all maximal ideals $P$ of the ring of integers of $F$ (this is essentially [3, Theorem 2.4]).

Specifically, let $(Y, h), h: Y \rightarrow \mathbb{A}^{m}$, be a principalisation of the ideal

$$
\mathcal{I}=\prod_{\kappa \in[k], \iota \in I_{\kappa}}\left(\mathbf{f}_{\kappa \iota}\right)
$$

where (f) denotes the ideal generated by the finite set $\mathbf{f}$ of polynomials. We set $\mathcal{V}:=\operatorname{Spec}(\mathrm{F}[\mathbf{y}] / \mathcal{I})$ and $\mathcal{V}_{\kappa \iota}:=\operatorname{Spec}\left(\mathrm{F}[\mathbf{y}] /\left(\mathbf{f}_{\kappa \iota}\right)\right)$. Then, denoting by $E_{t}$, $t \in T$, the irreducible components of $\left(h^{-1}(\mathcal{V})\right)_{\text {red }}$, we have

$$
\begin{aligned}
h^{-1}(\mathcal{V}) & =\sum_{t \in T} N_{t} E_{t}, \\
h^{-1}\left(\mathcal{V}_{\kappa \iota}\right) & =\sum_{t \in T} N_{t \kappa \iota} E_{t},
\end{aligned}
$$

say, for suitable non-negative integers $N_{t}, N_{t \kappa \iota}$. Note that, for every $t \in T$,

$$
N_{t}=\sum_{\kappa \in[k], \iota \in I_{\kappa}} N_{t \kappa \iota} .
$$

Similarly we denote by $\nu_{t}-1$ the multiplicity of $E_{t}$ in the divisor of $h^{*}\left(\mathrm{~d} y_{1} \wedge\right.$ $\left.\cdots \wedge \mathrm{d} y_{m}\right)$. The numbers $\left(N_{t \kappa \iota}, \nu_{t}\right)_{t \in T, \kappa \in[k], \iota \in I_{\kappa}}$ will be called the numerical data of the principalisation $(Y, h)$.

Theorem 2.1. Suppose that all the sets $\mathbf{f}_{\kappa \iota}$ are integral (i.e. contained in $R[\mathbf{y}])$ and do not define the zero ideal $\bmod P_{K}$, and that $(Y, h)$ has good reduction $\bmod P_{K}$. Then

$$
Z_{W, K, I}(\mathbf{s})=\frac{\left(1-q^{-1}\right)^{|I|}}{q^{m}} \sum_{U \subseteq T} c_{U, W}(q)(q-1)^{|U|} \Xi_{U, I}(q, \mathbf{s}),
$$

where

$$
c_{U, W}(q)=\mid\left\{a \in \bar{Y}(\bar{K}) \mid a \in \overline{E_{u}}(\bar{K}) \Leftrightarrow u \in U \text { and } \bar{h}(a) \in \bar{W}\right\} \mid
$$


(where ${ }^{-}$denotes reduction $\bmod P$ and $\bar{W}=\left\{\left(\overline{y_{1}}, \ldots, \overline{y_{m}}\right) \mid\left(y_{1}, \ldots, y_{m}\right) \in\right.$ $W\} \mid)$ and

(9)

$$
\Xi_{U, I}(q, \mathbf{s})=\sum_{\substack{\left(m_{u}\right)_{u \in U} \in \mathbb{N}^{|U|} \\\left(n_{i}\right)_{i \in I} \in \mathbb{N}^{l}}} q^{-\sum_{i} n_{i}-\sum_{u} \nu_{u} m_{u}-\sum_{\kappa} s_{\kappa} \min _{\iota \in I_{\kappa}}\left\{\sum_{i} e_{i \kappa \iota} n_{i}+\sum_{u} N_{u \kappa \iota} m_{u}\right\}} .
$$

Example 2.1. If $l=0$ and, for each $\kappa \in[k],\left|I_{\kappa}\right|=1$, Theorem 2.1 reduces to (a multivariable version of) Vey's and Zuniga-Galindo's generalisation [29, Theorem 2.10] to polynomial mappings of Denef's explicit formula [3, Theorem 2] for Igusa's local zeta function associated to a single polynomial. Notice in particular that in this case

$$
\Xi_{U, \varnothing}(q, \mathbf{s})=\sum_{\left(m_{u}\right)_{u \in U} \in \mathbb{N}|U|} q^{\sum_{u} m_{u}\left(-\nu_{u}-\sum_{\kappa} N_{u \kappa} s_{\kappa}\right)}=\prod_{u \in U} \frac{X_{u}}{1-X_{u}}
$$

for $X_{u}:=q^{-\nu_{u}-\sum_{\kappa} N_{u \kappa} s_{\kappa}}$, where we write $N_{u \kappa}$ for $N_{u \kappa \iota}$. Also compare Example 2.2 for the other 'extremal case' $\Xi_{\varnothing, I}(q, \mathbf{s})$.

Proof. The proof is analogous to the one of $[3$, Theorem 3.1] $(=[5$, Theorem $2]$ ), with the concept of resolution of singularities replaced by the concept of principalisation of ideals. We adopt - mutatis mutandis - Denef's notation and just explain how the proof differs from his. Let $a$ be a closed point of $\bar{Y}$, and thus also of $\widetilde{Y}$. Let $T_{a}=\left\{t \in T \mid a \in \overline{E_{t}}\right\}=\left\{t_{1}, \ldots, t_{r}\right\}_{<}$, say. Define $H=\left\{b \in Y(K) \mid h(b) \in R^{m}\right\}$ and recall the definition of the 'reduction $\bmod P^{\prime}$-map $\theta: H \rightarrow \bar{Y}(\bar{K})$. In the regular local ring $\mathcal{O}_{\widetilde{Y}, a}$, there exist irreducible elements $\gamma_{1}, \ldots, \gamma_{m}$ such that, on $\theta^{-1}(a)$, for all $\kappa \in[k], \iota \in I_{\kappa}$,

$$
\begin{aligned}
\left\|\mathbf{f}_{\kappa \iota} \circ h\right\| & =\left|\gamma_{1}\right|^{N_{t_{1} \kappa \iota}} \ldots\left|\gamma_{r}\right|^{N_{t_{r} \kappa \iota}} \text { and } \\
\left|h^{*}\left(\mathrm{~d} y_{1} \wedge \ldots \mathrm{d} y_{m}\right)\right| & =\left.\left|\gamma_{1}\right|^{\nu_{t_{1}}-1}|\ldots| \gamma_{r}\right|^{\nu_{t_{r}}-1}\left|\mathrm{~d} \gamma_{1} \wedge \ldots \wedge \mathrm{d} \gamma_{m}\right| .
\end{aligned}
$$

Setting $\mathrm{d} \gamma:=\mathrm{d} \gamma_{1} \wedge \cdots \wedge \mathrm{d} \gamma_{m}$ we define

$$
\begin{aligned}
& Z_{a, I}(\mathbf{s}) \\
& :=\int_{P^{l} \times \theta^{-1}(a)} \prod_{\kappa \in[k]} \max _{\iota \in I_{\kappa}}\left\{\prod_{i \in I}\left|x_{i}\right|^{e_{i \kappa \iota}} \prod_{\rho \in[r]}\left|\gamma_{\rho}\right|^{N_{t_{\rho} \kappa \iota}}\right\}^{s_{\kappa}} \prod_{\rho \in[r]}\left|\gamma_{\rho}\right|^{\nu_{t_{\rho}}-1}\left|\mathrm{~d} \mathbf{x}_{I}\right||\mathrm{d} \boldsymbol{\gamma}| \\
& =\int_{P^{l+m}} \prod_{\kappa \in[k]} \max _{\iota \in I_{\kappa}}\left\{\prod_{i \in I}\left|x_{i}\right|^{e_{i \kappa \iota}} \prod_{\rho \in[r]}\left|y_{\rho}\right|^{N_{t_{\rho} \kappa \iota}}\right\}^{s_{\kappa}} \prod_{\rho \in[r]}\left|y_{\rho}\right|^{\nu_{t_{\rho}}-1}\left|\mathrm{~d} \mathbf{x}_{I}\right||\mathrm{d} \mathbf{y}| \\
& =\frac{(q-1)^{r+l}}{q^{m+l}} \sum_{\substack{\left(m_{t}\right)_{t \in T_{a} \in \mathbb{N}^{r}} \\
\left(n_{i}\right)_{i \in I} \in \mathbb{N}^{l}}} q^{-\sum_{i} n_{i}-\sum_{t} \nu_{t} m_{t}-\sum_{\kappa} s_{\kappa} \min _{\iota \in I_{\kappa}}\left\{\sum_{i} e_{i \kappa \iota} n_{i}+\sum_{t} N_{t \kappa \iota} m_{t}\right\}} .
\end{aligned}
$$

This suffices as $Z_{W, K, I}(\mathbf{s})=\sum_{a \in \bar{Y}(\bar{K})} Z_{a, I}(\mathbf{s})$. 
We now make the further assumption that $m=n^{2}$. We identify $K^{n^{2}}$ with $\operatorname{Mat}_{n}(K)$ and assume that the ideals $\left(\mathbf{f}_{\kappa \iota}\right), \kappa \in[k], \iota \in I_{\kappa}$, are $B(F)$-invariant, where $B(F)$ is the group of $F$-rational points of the Borel subgroup of uppertriangular matrices in $G=\mathrm{GL}_{n}$, acting on $K\left[y_{11}, y_{12}, \ldots, y_{n n}\right]$ by matrixmultiplication from the right. Let $(Y, h), h: Y \rightarrow G / B$ be a principalisation of the ideal $\mathcal{I}=\prod_{\kappa, \iota}\left(\mathbf{f}_{\kappa \iota}\right)$. Denoting, similar to the above, by $\mathcal{V}$ the subvariety of $G / B(K)$ defined by $\mathcal{I}$ and by $\mathcal{V}_{\kappa \iota}$ the subvariety defined by $\left(\mathbf{f}_{\kappa \iota}\right)$ yields numerical data $\left(N_{t \kappa \iota}, \nu_{t}\right)_{t \in T, \kappa \in[k], \iota \in I_{\kappa}}$ defined by formulae analogous to (7) and (8) above. We shall study the integral

$$
Z_{I}(\mathbf{s}):=Z_{W, K, I}(\mathbf{s})
$$

for $W=\Gamma=\mathrm{GL}_{n}(R)$ for almost all completions $K$ of $F$. Note that the Haar measure $\mu^{\prime}$ on the compact topological group $\Gamma$ coincides with the additive Haar measure $\mu$ induced from $R^{n^{2}}$ (and normalised such that $\mu\left(R^{n^{2}}\right)=1$ ), as $\mu^{\prime}=|\operatorname{det}|^{-n} \mu=\mu$. This will be important in later applications as it implies, for example, that all the cosets of a finite index subgroup $\Gamma^{\prime} \leq \Gamma$ have measure $\mu(\Gamma) /\left|\Gamma: \Gamma^{\prime}\right|$, with $\mu(\Gamma)=\left(1-q^{-1}\right) \ldots\left(1-q^{-n}\right)$.

Theorem 2.2. Suppose that, in addition to the above assumptions, none of the ideals $\left(\mathbf{f}_{\kappa \iota}\right)$ is equal to the zero ideal $\bmod P_{K}$, and that $(Y, h)$ has good reduction $\bmod P_{K}$. Then

$$
Z_{I}(\mathbf{s})=\frac{\left(1-q^{-1}\right)^{|I|+n}}{q^{\left(\begin{array}{c}
n \\
2
\end{array}\right)}} \sum_{U \subseteq T} c_{U}(q)(q-1)^{|U|} \Xi_{U, I}(q, \mathbf{s}),
$$

where each $c_{U}(q)$ is the number of $\bar{K}$-rational points of $\overline{E_{U}} \backslash \cup_{V \supsetneq U} \overline{E_{V}}$ ( $E_{U}:=$ $\left.\cap_{u \in U} E_{u}\right)$ and $\Xi_{U, I}(q, \mathbf{s})$ is defined as in (9) above.

Proof. The proof follows closely the spirit of the proof of [5, Theorem 3]. In fact, our function $Z_{I}(\mathbf{s})$ is a close analogue of the function $\widehat{Z}_{K}(s)$, defined on $[5$, p. 1140]. We write $\Gamma$ as a disjoint union of sets

$$
\Gamma_{\sigma}=\left\{\mathbf{x} \in \Gamma \mid \overline{\mathbf{x}} \in B\left(\mathbb{F}_{q}\right) \sigma B\left(\mathbb{F}_{q}\right)\right\},
$$

$\sigma \in S_{n}$, where $\mathrm{GL}_{n}\left(\mathbb{F}_{q}\right)=\bigcup_{\sigma \in S_{n}} B\left(\mathbb{F}_{q}\right) \sigma B\left(\mathbb{F}_{q}\right)$ is the Bruhat decomposition (here $\sigma \in S_{n}$ is identified with the respective permutation matrix in $\mathrm{GL}_{n}\left(\mathbb{F}_{q}\right)$ ). Thus

$$
Z_{I}(\mathbf{s})=\sum_{\sigma \in S_{n}} Z_{\Gamma_{\sigma}, K, I}(\mathbf{s})
$$

There is an obvious map $\gamma: \Gamma \rightarrow G / B(K)$, and, by our invariance assumption on the ideals $\left(\mathbf{f}_{\kappa \iota}\right)$, the value of the integrand of $Z_{I}(\mathbf{s})$ at a point $(\mathbf{x}, \mathbf{y}) \in P^{l} \times \Gamma$ only depends on $\mathbf{x}$ and $\gamma(\mathbf{y})$. By taking the measure $\omega$ on $G / B(K)$ which induces the Haar measure on the unit ball $R^{\left(\begin{array}{l}n \\ 2\end{array}\right)}$ of each affine chart satisfying $\omega\left(a+P^{\left(\begin{array}{l}n \\ 2\end{array}\right)}\right)=q^{-\left(\begin{array}{c}n \\ 2\end{array}\right)}$ and noting that $\mu(B)=\left(1-q^{-1}\right)^{n}$, we obtain

$$
Z_{\Gamma_{\sigma}, K, I}(\mathbf{s})=\left(1-q^{-1}\right)^{n} \int_{P^{l} \times V_{\sigma}} \prod_{\kappa \in[k]}\left\|\mathbf{g}_{\kappa, I}(\mathbf{x}, \mathbf{y})\right\|^{s_{\kappa}}\left|\mathrm{d} \mathbf{x}_{I}\right| \mathrm{d} \omega
$$


where $V_{\sigma}=\gamma\left(\Gamma_{\sigma}\right)$. The projective variety $G / B$ may be covered by varieties $U_{\sigma}$, isomorphic to affine $\left(\begin{array}{l}n \\ 2\end{array}\right)$-space, indexed by the elements of the symmetric group $S_{n}$, such that each $V_{\sigma}$ is contained in $U_{\sigma}$ and is a union of cosets $\bmod P^{\left(\begin{array}{c}n \\ 2\end{array}\right)}$. Theorem 2.1 may thus be applied to the restriction $\left(Y^{\sigma}, h^{\sigma}\right)$ of $(Y, h)$, a principalisation of the ideal defining the restriction of $\mathcal{V}$ to $U_{\sigma}$ $\left(Y^{\sigma}=h^{-1}\left(U^{\sigma}\right), h^{\sigma}=\left.h\right|_{Y^{\sigma}}\right)$, with good reduction $\bmod P$. We obtain

$\int_{P^{l} \times V_{\sigma}} \prod_{\kappa \in[k]}\left\|\mathbf{g}_{\kappa, I}(\mathbf{x}, \mathbf{y})\right\|^{s_{\kappa}}\left|\mathrm{d} \mathbf{x}_{I}\right| \mathrm{d} \omega=\frac{\left(1-q^{-1}\right)^{l}}{q^{\left(\begin{array}{c}n \\ 2\end{array}\right)}} \sum_{U \subseteq T} c_{U, \sigma}(q)(q-1)^{|U|} \Xi_{U, I}(q, \mathbf{s})$,

where

$$
c_{U, \sigma}(q)=\mid\left\{a \in \bar{Y}^{\sigma}(\bar{K}) \mid a \in \overline{E_{u}}(\bar{K}) \Leftrightarrow u \in U \text { and } \bar{h}(a) \in \overline{V_{\sigma}}\right\} \mid .
$$

The result follows since, if $a \in \bar{Y}(\bar{K})$, then $\bar{h}(a)$ is in exactly one $\overline{V_{\sigma}}$. Thus $\sum_{\sigma \in S_{n}} c_{U, \sigma}(q)=\left|\left\{a \in \bar{Y}(\bar{K}) \mid a \in \overline{E_{u}}(\bar{K}) \Leftrightarrow u \in U\right\}\right|=c_{U}(q)$.

We now consider the normalised integrals

$$
\widetilde{Z_{I}}(\mathbf{s}):=\frac{Z_{I}(\mathbf{s})}{\left(1-q^{-1}\right)^{|I|} \mu(\Gamma)} .
$$

Corollary 2.1. For $U \subseteq T$, let $b_{U}(q)$ denote the number of $\bar{K}$-rational points of $\overline{E_{U}}$. Then

$$
\widetilde{Z_{I}}(\mathbf{s})=\left|G / B\left(\mathbb{F}_{q}\right)\right|^{-1} \sum_{U \subseteq T} b_{U}(q) \sum_{V \subseteq U}(-1)^{|U \backslash V|}(q-1)^{|V|} \Xi_{V, I}(q, \mathbf{s}) .
$$

Proof. This follows immediately from the formula given for $Z_{I}(\mathbf{s})$ in Theorem 2.2, Definition (10), the fact that $\left|G / B\left(\mathbb{F}_{q}\right)\right|=\left(\begin{array}{c}n \\ {[n-1]}\end{array}\right)_{q}$ and from the identity

$$
c_{V}(q)=\sum_{V \subseteq U \subseteq T}(-1)^{|U \backslash V|} b_{U}(q)
$$

Before we proceed we consider a very special case.

Example 2.2. Assume that, for all $\kappa \in[k],\left|I_{\kappa}\right|=1$ and that all $\mathbf{f}_{\kappa \iota}=\{1\}$. We write $e_{i \kappa}$ for $e_{i \kappa \iota}$. We have

$$
\Xi_{\varnothing, I}(q, \mathbf{s})=\sum_{\left(n_{i}\right)_{i \in I} \in \mathbb{N}^{l}} q^{\sum_{i} n_{i}\left(-1-\sum_{\kappa} e_{i \kappa} s_{\kappa}\right)}=\prod_{i \in I} \frac{X_{i}}{1-X_{i}},
$$

with $X_{i}=q^{-1-\sum_{\kappa} e_{i \kappa} s_{\kappa}}$. Also note that $b_{\varnothing}(q)=\left|G / B\left(\mathbb{F}_{q}\right)\right|$ and that $b_{U}(q)=$ 0 if $U \neq \varnothing$. Thus $\widetilde{Z_{I}}(\mathbf{s})=\prod_{i \in I} \frac{X_{i}}{1-X_{i}}$. It is trivial to verify the 'inversion property'

$$
\left.\widetilde{Z_{I}}(\mathbf{s})\right|_{q \rightarrow q^{-1}}=(-1)^{|I|} \sum_{J \subseteq I} \widetilde{Z_{J}}(\mathbf{s})
$$


as

$$
\prod_{i \in I} \frac{X_{i}^{-1}}{1-X_{i}^{-1}}=(-1)^{|I|} \sum_{J \subseteq I} \prod_{j \in J} \frac{X_{j}}{1-X_{j}} .
$$

In the remainder of the current section we shall show that equation (12) holds under the premises of Theorem 2.2. To give meaning to the left-most term in (12) in general we have to explain what we mean by $b_{U}\left(q^{-1}\right)$ (the other constituents of the expression $(11)$ for $\widetilde{Z_{I}}(\mathbf{s})$ being rational functions in $q$ and $\left.q^{-s_{1}}, \ldots, q^{-s_{k}}\right)$. Recall that by properties of the Weil zeta functions associated to the $\left(\left(\begin{array}{l}n \\ 2\end{array}\right)-|U|\right)$-dimensional smooth projective varieties $\overline{E_{U}}$ it is known that

$$
b_{U}(q)=\sum_{r=0}^{2\left(\left(\begin{array}{c}
n \\
2
\end{array}\right)-|U|\right)}(-1)^{r} \sum_{j=1}^{t_{U, r}} \alpha_{U, r, j}
$$

for suitable non-negative integers $t_{U, r}$ and non-zero complex numbers $\alpha_{U, r, j}$, with the property that, for each $U, r$, the multisets

$$
\left\{\alpha_{U, r, j} \mid j \in\left[t_{U, 2\left(\left(\begin{array}{l}
n \\
2
\end{array}\right)-|U|\right)-r}\right]\right\}
$$

and

$$
\left\{\frac{q^{\left(\begin{array}{c}
n \\
2
\end{array}\right)-|U|}}{\alpha_{U, r, j}} \mid j \in\left[t_{U, r}\right]\right\}
$$

coincide (cf., e.g., [5, Proof of Theorem 4]). This motivates the definition

$$
b_{U}\left(q^{-1}\right):=q^{-\left(\left(\begin{array}{l}
n \\
2
\end{array}\right)-|U|\right)} b_{U}(q)=\sum_{r=0}^{\left.2\left(\begin{array}{c}
n \\
2
\end{array}\right)-|U|\right)}(-1)^{r} \sum_{j=1}^{t_{U, r}} \alpha_{U, r, j}^{-1} .
$$

We shall prove

Theorem 2.3. Under the assumptions of Theorem 2.2, the following 'inversion properties' hold:

$$
\forall I \subseteq[n-1]:\left.\widetilde{Z_{I}}(\mathbf{s})\right|_{q \rightarrow q^{-1}}=(-1)^{|I|} \sum_{J \subseteq I} \widetilde{Z_{J}}(\mathbf{s})
$$

Proof. To see what happens to the (rational) functions $\Xi_{V, I}(q, \mathbf{s})$ in expression (11) if we formally invert the prime power $q$, we employ a result of Stanley:

Proposition 2.1. Let $L_{\sigma \tau}(\mathbf{n}), \sigma \in[s], \tau \in[t]$, be $\mathbb{Z}$-linear forms in the variables $n_{1}, \ldots, n_{r}$ and $X_{1}, \ldots, X_{r}, Y_{1}, \ldots, Y_{s}$ independent variables, and set

$$
\begin{aligned}
Z^{\circ}(\mathbf{X}, \mathbf{Y}) & :=\sum_{\mathbf{n} \in \mathbb{N}^{r}} \prod_{\rho \in[r]} X_{\rho}^{n_{\rho}} \prod_{\sigma \in[s]} Y_{\sigma}^{\min _{\tau \in[t]}\left\{L_{\sigma \tau}(\mathbf{n})\right\}} \\
Z(\mathbf{X}, \mathbf{Y}) & :=\sum_{\mathbf{n} \in \mathbb{N}_{0}^{r}} \prod_{\rho \in[r]} X_{\rho}^{n_{\rho}} \prod_{\sigma \in[s]} Y_{\sigma}^{\min _{\tau \in[t]}\left\{L_{\sigma \tau}(\mathbf{n})\right\}}
\end{aligned}
$$


Then

$$
Z^{\circ}\left(\mathbf{X}^{-1}, \mathbf{Y}^{-1}\right)=(-1)^{r} Z(\mathbf{X}, \mathbf{Y}) .
$$

Proof. The proof of [28, Theorem 4.6.14] carries through to this slightly more general situation, provided one chooses a triangulation of $\mathbb{N}^{r}$ that refines a subdivision into rational polyhedral cones eliminating the 'min'-terms in the sum defining $Z(\mathbf{X}, \mathbf{Y})$.

Corollary 2.2. For all $I \subseteq[n-1], V \subseteq T$,

$$
\left.\Xi_{V, I}(q, \mathbf{s})\right|_{q \rightarrow q^{-1}}=(-1)^{|V|+|I|} \sum_{W \subseteq V, J \subseteq I} \Xi_{W, J}(q, \mathbf{s}) .
$$

We record the following simple fact:

Lemma 2.1. For all $U \subseteq T, J \subseteq[n-1]$,

$$
\begin{aligned}
& \sum_{V \subseteq U}(-1)^{|U \backslash V|}\left(1-q^{-1}\right)^{|V|} \sum_{W \subseteq V} \Xi_{W, J}(q, \mathbf{s})= \\
& q^{-|U|} \sum_{V \subseteq U}(-1)^{|U \backslash V|}(q-1)^{|V|} \Xi_{V, J}(q, \mathbf{s}) .
\end{aligned}
$$

The proof is a simple computation. We can now deduce

$$
\begin{aligned}
\left.\widetilde{Z_{I}}(\mathbf{s})\right|_{q \rightarrow q^{-1}}= & \frac{(-1)^{|I|} q^{\left(\begin{array}{c}
n \\
2
\end{array}\right)}}{\left|G / B\left(\mathbb{F}_{q}\right)\right|} \sum_{U \subseteq T} b_{U}\left(q^{-1}\right) \sum_{V \subseteq U}(-1)^{|U \backslash V|}\left(1-q^{-1}\right)^{|V|} . \\
& \sum_{W \subseteq V, J \subseteq I} \Xi_{W, J}(q, \mathbf{s}) \\
= & (-1)^{|I|} \sum_{J \subseteq I}\left|G / B\left(\mathbb{F}_{q}\right)\right|^{-1} \sum_{U \subseteq T} q^{|U|} b_{U}(q) . \\
& \sum_{V \subseteq U}(-1)^{|U \backslash V|}\left(1-q^{-1}\right)^{|V|} \sum_{W \subseteq V} \Xi_{W, J}(q, \mathbf{s}) \\
& \sum_{J \subseteq I}\left|G / B\left(\mathbb{F}_{q}\right)\right|^{-1} \sum_{U \subseteq T} b_{U}(q) . \\
& =(-1)^{|I|} \sum_{J \subseteq U} \widetilde{Z_{J}}(\mathbf{s}) .
\end{aligned}
$$

This completes the proof of Theorem 2.3.

Recall that the polynomials $\left(\begin{array}{l}n \\ I\end{array}\right)_{X}$ were introduced at the end of the introduction. We define

$$
\widetilde{Z}(\mathbf{s})=\sum_{I \subseteq[n-1]}\left(\begin{array}{l}
n \\
I
\end{array}\right)_{q^{-1}} \widetilde{Z_{I}}(\mathbf{s}) .
$$

To prove Theorems A, B and C we shall need 
Corollary 2.3. Under the assumptions of Theorem 2.2, the following functional equation holds:

$$
\left.\widetilde{Z}(\mathbf{s})\right|_{q \rightarrow q^{-1}}=(-1)^{n-1} q^{\left(\begin{array}{c}
n \\
2
\end{array}\right)} \widetilde{Z}(\mathbf{s}) .
$$

Proof. This follows from the proof of [32, Corollary 2]. Note that Theorem 2.2 provides the required analogue of [32, Lemma 6].

Theorem D will follow from Proposition 2.2 of the next section which is in turn a special case of the following straightforward corollary.

Corollary 2.4. Under the assumptions of Theorem 2.2, for any $i \in[n-1]$,

$$
\left.\left(\widetilde{Z_{\varnothing}}(\mathbf{s})+\left(1-q^{-n}\right) \widetilde{Z_{\{i\}}}(\mathbf{s})\right)\right|_{q \rightarrow q^{-1}}=q^{n}\left(\widetilde{Z_{\varnothing}}(\mathbf{s})+\left(1-q^{-n}\right) \widetilde{Z_{\{i\}}}(\mathbf{s})\right) .
$$

2.2. A first application: Counting elementary divisors. We show how the problem of counting elementary divisors of matrices of forms may be reduced to the problem of computing $p$-adic integrals of the form studied in the previous section, associated to the polynomials describing the degeneracy loci of these matrices. The main result of this subsection - Proposition $2.2-$ will be needed to prove Theorem D in Section 3.4.

Again, let $K$ be a $p$-adic field with valuation ring $R$, whose maximal ideal is denoted by $P$. Let $\mathcal{R}$ be an $e \times f$-matrix (with $e \geq f$, say) of polynomials $\mathcal{R}_{i j}(\mathbf{Y}) \in R\left[Y_{1}, \ldots, Y_{n}\right]$. We make the assumption on $\mathcal{R}$ that, whenever $\mathbf{y}=\left(y_{1}, \ldots, y_{n}\right) \in R^{n}$ is a vector with $\overline{\mathbf{y}} \neq 0$, at least one entry of $\overline{\mathcal{R}(\mathbf{y})}$ is non-zero. For a non-negative integer $N$ and a vector y $P^{N} \in\left(R / P^{N}\right)^{n}$ we say that $\mathcal{R}\left(\mathbf{y} P^{N}\right)$ has elementary divisor type $\mathbf{m}\left(\right.$ written $\left.\nu\left(\mathcal{R}\left(\mathbf{y} P^{N}\right)\right)=\mathbf{m}\right)$ if $\mathbf{m}=\left(m_{1}, \ldots, m_{f}\right), m_{i} \in[0, N], m_{1} \leq \cdots \leq m_{f}$, and there are matrices $\beta \in \mathrm{GL}_{e}\left(R / P^{N}\right), \gamma \in \mathrm{GL}_{f}\left(R / P^{N}\right)$ such that

$$
\beta \mathcal{R}\left(\mathbf{y} P^{N}\right) \gamma \equiv\left(\begin{array}{ccc}
q^{m_{1}} & & \\
& \ddots & \\
& & q^{m_{f}}
\end{array}\right) \quad \bmod P^{N} .
$$

For $\mathbf{m} \in \mathbb{N}_{0}^{f}$ we set

$$
\mathcal{N}_{N, \mathcal{R}, \mathbf{m}}:=\left|\left\{\mathbf{y} P^{N} \in\left(R / P^{N}\right)^{n} \mid \overline{\mathbf{y} P^{N}} \neq 0, \nu\left(\mathcal{R}\left(\mathbf{y} P^{N}\right)\right)=\mathbf{m}\right\}\right| .
$$

Note that $\mathcal{N}_{N, \mathcal{R}, \mathbf{m}}=0$ unless $0=m_{1} \leq \cdots \leq m_{f} \leq N$ (the necessity of $m_{1}=0$ being a consequence of our assumption on $\mathcal{R}$ ). Given, in addition, a $g \times h$-submatrix $\mathcal{S}$ of $\mathcal{R}$ (WLOG $g \geq h$ ) defined by choosing $g$ rows and $h$ columns of $\mathcal{R}$, and an $h$-tuple $\mathbf{n}$ we define

$$
\mathcal{N}_{N, \mathcal{R}, \mathcal{S}, \mathbf{m}, \mathbf{n}}:=\left|\left\{\mathbf{y} P^{N} \in\left(R / P^{N}\right)^{n} \mid \overline{\mathbf{y}} \neq 0, \begin{array}{c}
\nu\left(\mathcal{R}\left(\mathbf{y} P^{N}\right)\right)=\mathbf{m}, \\
\nu\left(\mathcal{S}\left(\mathbf{y} P^{N}\right)\right)=\mathbf{n}
\end{array}\right\}\right| .
$$

Again, $\mathcal{N}_{N, \mathcal{R}, \mathcal{S}, \mathbf{m}, \mathbf{n}}=0$ unless $0=m_{1} \leq \cdots \leq m_{f} \leq N$ and $n_{1} \leq \cdots \leq n_{h}$. We suppress the subscripts $\mathcal{R}$ and $\mathcal{S}$ if they are clear from the context. 
Given complex variables $r_{1}, \ldots, r_{f}, s_{1}, \ldots, s_{h}$, we define the generating function

$$
P(\mathbf{r}, \mathbf{s})=P_{\mathcal{R}, \mathcal{S}, K}(\mathbf{r}, \mathbf{s})=\sum_{\substack{N \in \mathbb{N}_{0} \\ \mathbf{m} \in \mathbb{N}_{0}^{f}, \mathbf{n} \in \mathbb{N}_{0}^{h}}} \mathcal{N}_{N, \mathbf{m}, \mathbf{n}} q^{-\sum_{i \in[f]}\left(N-m_{i}\right) r_{i}-\sum_{j \in[h]}\left(N-n_{j}\right) s_{j}} .
$$

We now assume that the matrix $\mathcal{R}$ is in fact defined over a number field $F$, that its entries are all homogeneous of the same degree and that the above assumption on $\mathcal{R}$ is satisfied for almost all completions $K$ of $F$ for which all $\mathcal{R}_{i j}(\mathbf{Y}) \in R_{K}[\mathbf{Y}]$. We consider such a 'good' completion $K$ and drop the subscript $K$. For $i \in[f]_{0}$, let $\boldsymbol{\rho}_{i}$ denote the set of $i$-minors of $\mathcal{R}$. The polynomials $\boldsymbol{\rho}_{i}$ define the (rk $\leq i-1$ )-locus (or $i$-th degeneracy locus) of $\mathcal{R}(\mathbf{Y})$. Similarly, let $\boldsymbol{\sigma}_{j}, j \in[h]_{0}$, denote the set of $j$-minors of $\mathcal{S}$. Let

$$
k:=\max \left\{i \in[f]_{0} \mid\left(\boldsymbol{\rho}_{i}\right) \neq(0)\right\} \quad \text { and } \quad l:=\max \left\{j \in[h]_{0} \mid\left(\boldsymbol{\sigma}_{j}\right) \neq(0)\right\} .
$$

Note firstly that $\rho_{0}=\sigma_{0}=\{1\}$, secondly that, by our assumption on $\mathcal{R}$, $k \geq 1$, thirdly that $0 \leq l \leq k$ and fourthly that $P(\mathbf{r}, \mathbf{s})$ is really a function in the variables $r_{1}, \ldots, r_{k}, s_{1}, \ldots, s_{l}$ :

$$
P(\mathbf{r}, \mathbf{s})=\sum_{\substack{N \in \mathbb{N}_{0} \\ \mathbf{m} \in \mathbb{N}_{0}^{k}, \mathbf{n} \in \mathbb{N}_{0}^{l}}} \mathcal{N}_{N, \mathbf{m}, \mathbf{n}} q^{-\sum_{\kappa \in[k]}\left(N-m_{\kappa}\right) r_{\kappa}-\sum_{\lambda \in[l]}\left(N-n_{\lambda}\right) s_{\lambda}}
$$

where we set, given $\mathbf{m}=\left(m_{1}, \ldots, m_{k}\right)$ and $\mathbf{n}=\left(n_{1}, \ldots, n_{l}\right)$,

$$
\mathcal{N}_{N, \mathbf{m}, \mathbf{n}}:=\mathcal{N}_{N,\left(m_{1}, \ldots, m_{k}, N, \ldots, N\right),\left(n_{1}, \ldots, n_{l}, N, \ldots, N\right)} .
$$

For $I \subseteq\{1\}$ and $W=\Gamma=\mathrm{GL}_{n}(R)$ as above, consider the $p$-adic integral

$$
\begin{aligned}
Z_{I}(\mathbf{r}, \widetilde{\mathbf{r}}, \mathbf{s}, \widetilde{\mathbf{s}}, t):= & \int_{P|I| \times \Gamma}\left|x^{|I|}\right|^{t} \prod_{\kappa \in[k]}\left\|\boldsymbol{\rho}_{\kappa}\left(\mathbf{y}^{1}\right) \cup x^{|I|} \boldsymbol{\rho}_{\kappa-1}\left(\mathbf{y}^{1}\right)\right\|^{r_{\kappa}}\left\|\boldsymbol{\rho}_{\kappa-1}\left(\mathbf{y}^{1}\right)\right\|^{\widetilde{r}_{\kappa}} \\
& \prod_{\lambda \in[l]}\left\|\boldsymbol{\sigma}_{\lambda}\left(\mathbf{y}^{1}\right) \cup x^{|I|} \boldsymbol{\sigma}_{\lambda-1}\left(\mathbf{y}^{1}\right)\right\|^{s_{\lambda}}\left\|\boldsymbol{\sigma}_{\lambda-1}\left(\mathbf{y}^{1}\right)\right\|^{\widetilde{s}_{\lambda}}\left|\mathrm{d} x_{I} \| \mathrm{d} \mathbf{y}\right|,
\end{aligned}
$$

where $\mathbf{y}^{1}$ denotes the first column of the matrix $\mathbf{y} \in \Gamma$.

Remark 2.1. Whilst artificial, the formulation of $Z_{I}$ as an integral over $P^{|I|} \times \Gamma$ rather than over $P^{|I|} \times R^{n} \backslash P^{n}$ serves to make it fit the 'blueprint' Theorem 2.2 provided in the previous section.

We now set, for $\mathbf{m}=\left(m_{1}, \ldots, m_{k}\right) \in \mathbb{N}_{0}^{k}, \mathbf{n}=\left(n_{1}, \ldots, n_{l}\right) \in \mathbb{N}_{0}^{l}$ and $N \in \mathbb{N}$ $\mu_{N, \mathbf{m}, \mathbf{n}}=\mu\left\{(x, \mathbf{y}) \in P \times \Gamma \mid v(x)=N, \nu\left(\mathcal{R}\left(\mathbf{y}^{1} P^{N}\right)\right)=\mathbf{m}, \nu\left(\mathcal{S}\left(\mathbf{y}^{1} P^{N}\right)\right)=\mathbf{n}\right\}$ and $Z_{I}(\mathbf{r}, \mathbf{s}, t):=Z_{I}(\mathbf{r},-\mathbf{r}, \mathbf{s},-\mathbf{s}, t)$. Note that, by definition, $\mu_{N, \mathbf{m}, \mathbf{n}}=0$ unless $0 \leq m_{1} \leq \cdots \leq m_{k} \leq N$ and $n_{1} \leq \cdots \leq n_{l} \leq N$. By definition of the 
polynomials $\boldsymbol{\rho}_{\kappa}, \boldsymbol{\sigma}_{\lambda}$, we have

$$
\begin{aligned}
Z_{\varnothing}(\mathbf{r}, \mathbf{s}, t) & =\mu(\Gamma) \quad\left(\text { and thus, by }(10), \widetilde{Z_{\varnothing}}(\mathbf{r}, \mathbf{s}, t)=1\right), \\
Z_{\{1\}}(\mathbf{r}, \mathbf{s}, t) & =\sum_{\substack{N \in \mathbb{N} \\
\mathbf{m} \in \mathbb{N}_{0}^{k}, \mathbf{n} \in \mathbb{N}_{0}^{l}}} \mu_{N, \mathbf{m}, \mathbf{n}} q^{-t N-\sum_{\kappa} r_{\kappa} m_{\kappa}-\sum_{\lambda} s_{\lambda} n_{\lambda}} .
\end{aligned}
$$

Theorem 2.2 is applicable to $Z_{I}, I \subseteq\{1\}$, together with a principalisation $(Y, h), h: Y \rightarrow G / B$, of the ideal $\mathcal{I}=\prod_{\kappa \in[k]}\left(\boldsymbol{\rho}_{\kappa}\right) \prod_{\lambda \in[l]}\left(\boldsymbol{\sigma}_{\lambda}\right)$. Indeed, $B(F)$ invariance is a consequence of the fact that the entries of $\mathcal{R}$ were all assumed to be homogeneous of the same degree.

The following crucial lemma relates the numbers $\mu_{N, \mathbf{m}, \mathbf{n}}$ with the data $\mathcal{N}_{N, \mathbf{m}, \mathbf{n}}$ we would like to capture:

Lemma 2.2. For $N \in \mathbb{N}$

$$
\mathcal{N}_{N, \mathbf{m}, \mathbf{n}}=\frac{\left(\begin{array}{l}
n \\
1
\end{array}\right)_{q^{-1}}}{\mu(\Gamma)} \mu_{N, \mathbf{m}, \mathbf{n}} q^{N(n+1)} .
$$

Recall that $\left(\begin{array}{l}n \\ 1\end{array}\right)_{q^{-1}}=\left(1-q^{-n}\right) /\left(1-q^{-1}\right)$.

Proof. Let $\Gamma_{\{1\}, N}$ denote the group

$$
\left\{\left(\begin{array}{c|c}
\gamma_{1} & * \\
\hline q^{N} * & \gamma_{n-1}
\end{array}\right)\right\}
$$

where $\gamma_{i}$ stands for a matrix in $\Gamma_{i}=\mathrm{GL}_{i}(R)$, * for an arbitrary matrix with entries in $R$, and $q^{N} *$ for a matrix of the appropriate size with entries in $q^{N} R$, respectively. Then the set

$$
\left\{(x, \mathbf{y}) \in P \times \Gamma \mid v(x)=N, \nu\left(\mathcal{R}\left(\mathbf{y}^{1} P^{N}\right)\right)=\mathbf{m}, \nu\left(\mathcal{S}\left(\mathbf{y}^{1} P^{N}\right)\right)=\mathbf{n}\right\}
$$

may be written as a disjoint union of the $\left|\Gamma: \Gamma_{\{1\}, N}\right|$ sets

$$
\left\{(x, \mathbf{y}) \in P \times \gamma \Gamma_{\{1\}, N} \mid v(x)=N, \nu\left(\mathcal{R}\left(\mathbf{y}^{1} P^{N}\right)\right)=\mathbf{m}, \nu\left(\mathcal{S}\left(\mathbf{y}^{1} P^{N}\right)\right)=\mathbf{n}\right\}
$$

where $\gamma$ runs through a complete set of coset representatives of $\Gamma / \Gamma_{\{1\}, N}$. The measure of each of these sets is either zero or equals $\left(1-q^{-1}\right) q^{-N} \mu\left(\Gamma_{\{1\}, N}\right)$. The latter happens $\mathcal{N}_{N, \mathbf{m}, \mathbf{n}}^{\prime}$ times, where

$$
\mathcal{N}_{N, \mathbf{m}, \mathbf{n}}^{\prime}=\left|\left\{\mathbf{y}^{1} \in \mathbb{P}^{n-1}\left(R / P^{N}\right) \mid \overline{\mathbf{y}^{1}} \neq 0, \nu\left(\mathcal{R}\left(\mathbf{y}^{1}\right)\right)=\mathbf{m}, \nu\left(\mathcal{S}\left(\mathbf{y}^{1}\right)\right)=\mathbf{n}\right\}\right| .
$$

Clearly $\mathcal{N}_{N, \mathbf{m}, \mathbf{n}}=\left(1-q^{-1}\right) q^{N} \mathcal{N}_{N, \mathbf{m}, \mathbf{n}}^{\prime}$. Using the identity

$$
\mu(\Gamma) / \mu\left(\Gamma_{\{1\}, N}\right)=\left(\begin{array}{c}
n \\
1
\end{array}\right)_{q^{-1}} q^{N(n-1)}
$$

we obtain

$$
\mu_{N, \mathbf{m}, \mathbf{n}}=\mathcal{N}_{N, \mathbf{m}, \mathbf{n}}^{\prime}\left(1-q^{-1}\right) q^{-N} \mu\left(\Gamma_{\{1\}, N}\right)=\mathcal{N}_{N, \mathbf{m}, \mathbf{n}} \frac{\mu(\Gamma)}{\left(\begin{array}{l}
n \\
1
\end{array}\right)_{q^{-1}} q^{N(n+1)}}
$$

as claimed. 
Lemma 2.2 yields

$$
\begin{aligned}
P(\mathbf{r}, \mathbf{s})-1= & P(\mathbf{r}, \mathbf{s})-\widetilde{Z_{\varnothing}}\left(-\mathbf{r},-\mathbf{s}, \sum_{\kappa} r_{\kappa}+\sum_{\lambda} s_{\lambda}-n-1\right) \\
= & \sum_{\substack{N \in \mathbb{N} \\
\mathbf{m} \in \mathbb{N}_{0}^{k}, \mathbf{n} \in \mathbb{N}_{0}^{l}}} \mathcal{N}_{N, \mathbf{m}, \mathbf{n}} q^{-\sum_{\kappa \in[k]}\left(N-m_{\kappa}\right) r_{\kappa}-\sum_{\lambda \in[l]}\left(N-n_{\lambda}\right) s_{\lambda}} \\
= & \frac{\left(\begin{array}{l}
n \\
1
\end{array}\right)_{q^{-1}}}{\mu(\Gamma)} \\
& \sum_{\substack{N \in \mathbb{N} \\
\mathbf{m} \in \mathbb{N}_{0}^{f}, \mathbf{n} \in \mathbb{N}_{0}^{h}}} \mu_{N, \mathbf{m}, \mathbf{n}} q^{N\left(n+1-\sum_{\kappa} r_{\kappa}-\sum_{\lambda} s_{\lambda}\right)+\sum_{\kappa} m_{\kappa} r_{\kappa}+\sum_{\lambda} n_{\lambda} s_{\lambda}} \\
= & \frac{\left(\begin{array}{l}
n \\
1
\end{array}\right)_{q^{-1}}}{\mu(\Gamma)} Z_{\{1\}}\left(-\mathbf{r},-\mathbf{s}, \sum_{\kappa} r_{\kappa}+\sum_{\lambda} s_{\lambda}-n-1\right) \\
= & \left(1-q^{-n}\right) \widetilde{Z_{\{1\}}}\left(-\mathbf{r},-\mathbf{s}, \sum_{\kappa} r_{\kappa}+\sum_{\lambda} s_{\lambda}-n-1\right) .
\end{aligned}
$$

From Corollary 2.4 we deduce

Proposition 2.2. For all but finitely many completions $K$ of $F$, the following functional equation holds:

$$
\left.P_{\mathcal{R}, \mathcal{S}, K}(\mathbf{r}, \mathbf{s})\right|_{q \rightarrow q^{-1}}=q^{n} P_{\mathcal{R}, \mathcal{S}, K}(\mathbf{r}, \mathbf{s}) .
$$

\section{Applications to ZETA FUnCtions OF GROUPS AND RINGS}

3.1. Zeta functions of rings. In this section we prove Theorem A. Let $L$ be a ring of torsion-free rank $n$. In fact, without loss of generality we may assume that $L$ is additively isomorphic to $\mathbb{Z}^{n}$. Let $p$ be a prime. Multiplication in $L$ is a bi-additive mapping $\beta: \mathbb{Z}^{n} \times \mathbb{Z}^{n} \rightarrow \mathbb{Z}^{n}$, which extends to $\beta_{p}: \mathbb{Z}_{p}^{n} \times \mathbb{Z}_{p}^{n} \rightarrow \mathbb{Z}_{p}^{n}$, inducing a $\mathbb{Z}_{p}$-algebra structure on $L_{p}:=L \otimes \mathbb{Z}_{p}$. We shall give a formula for the local zeta functions

$$
\zeta_{L, p}(s)=\sum_{H \leq L_{p}}\left|L_{p}: H\right|^{-s},
$$

where $H$ runs over the subalgebras of finite index in $L_{p}$, valid for almost all primes $p$, in terms of the $p$-adic integrals studied in Section 2. More precisely, we shall show that $\zeta_{L, p}(s)$ is expressible in terms of functions $\widetilde{Z}(\mathbf{s})$, defined as in (16), to which Corollary 2.3 is applicable.

Write $L=\mathbb{Z} l_{1} \oplus \cdots \oplus \mathbb{Z} l_{n}$. We consider the $n \times n$-matrix of $\mathbb{Z}$-linear forms

$$
\mathcal{R}(\mathbf{y})=\left(L_{i j}(\mathbf{y})\right) \in \operatorname{Mat}_{n}(\mathbb{Z}[\mathbf{y}]),
$$

where $L_{i j}(\mathbf{y}):=\sum_{k \in[n]} \lambda_{i j}^{k} y_{k}$, encoding the structure constants $\lambda_{i j}^{k}$ of $L$ with respect to the chosen basis, that is $l_{i} l_{j}=\sum_{k \in[n]} \lambda_{i j}^{k} l_{k}$. Let $C_{i}$ denote the matrix of the linear map given by right-multiplication with the generator $l_{i}$.

A full sublattice $\Lambda$ in $\left(L_{p},+\right)$ corresponds to a coset $\Gamma M$, where $\Gamma=\Gamma_{n}=$ $\mathrm{GL}_{n}\left(\mathbb{Z}_{p}\right)$ and the rows of the matrix $M=\left(m_{i j}\right) \in \mathrm{GL}_{n}\left(\mathbb{Q}_{p}\right) \cap \operatorname{Mat}_{n}\left(\mathbb{Z}_{p}\right)$, 
the set of integral $n \times n$-matrices with non-zero determinant, encode the coordinates of generators for $\Lambda$ with respect to the chosen basis. Denote by $M_{i}$ the $i$-th row of $M$. It is not hard to check (cf. the proof of [8, Theorem 5.5]) that $\Lambda$ is a $\mathbb{Z}_{p}$-subalgebra of $L_{p}$ if and only if

$$
\forall i, j \in[n]: M_{i} \sum_{r \in[n]} C_{r} m_{j r} \in\left\langle M_{k} \mid k \in[n]\right\rangle_{\mathbb{Z}_{p}} .
$$

Rather than trying to analyse the restrictions condition (24) imposes on the entries of suitable upper-triangular representatives of the coset $\Gamma M$ as in [8], we base our analysis on the following two basic observations.

The first point is that every homothety class of lattices $\Lambda$ in $\left(L_{p},+\right)$ contains a largest subalgebra $\Lambda_{0}$, and the subalgebras in this class are exactly the multiples $p^{m} \Lambda_{0}, m \in \mathbb{N}_{0}$. We thus have

$$
\zeta_{L, p}(s)=\left(1-p^{-n s}\right)^{-1} \sum_{[\Lambda]}\left|L_{p}: \Lambda_{0}\right|^{-s},
$$

where $\Lambda_{0}$ denotes the largest subalgebra in the homothety class $[\Lambda]=\left[\Lambda_{0}\right]$.

The second observation is that it is easy to check condition (24) if $M$ happens to be a diagonal matrix. With respect to the given basis, $\Gamma M$ may not admit a diagonal representative. By the elementary divisor theorem, however, it does contain a representative of the form $M=D \alpha^{-1}$, where $\alpha \in \Gamma$,

$$
D=D\left(I, \mathbf{r}_{0}\right)=p^{r_{0}} \operatorname{diag}(\underbrace{\underbrace{\sum_{\iota \in I} r_{\iota}}_{i_{1}}, \ldots, p^{\sum_{\iota \in I} r_{\iota}}}_{i_{l}}, \ldots, p^{r_{i_{l}}}, \ldots, p^{r_{i_{l}}}, 1, \ldots, 1)
$$

for a set $I=\left\{i_{1}, \ldots, i_{l}\right\}_{<} \subseteq[n-1]$ and a vector $\left(r_{0}, r_{i_{1}}, \ldots, r_{i_{l}}\right)=: \mathbf{r}_{0} \in \mathbb{N}_{0} \times \mathbb{N}^{l}$ (both depending only on $\Gamma M)$. We say that $\Lambda$ has type $\left(I, \mathbf{r}_{0}\right)$. We call $\Lambda$ maximal (in its homothety class) if $r_{0}=0$. We say that the homothety class $[\Lambda]$ has type $(I, \mathbf{r}), \mathbf{r}=\left(r_{i_{1}}, \ldots, r_{i_{l}}\right) \in \mathbb{N}^{l}$, - written $\nu([\Lambda])=(I, \mathbf{r})-$ if its maximal element has type $\left(I,\left(0, r_{i_{1}}, \ldots, r_{i_{l}}\right)\right)$. By slight abuse of notation we may also say that a lattice $\Lambda$ has type $I$ if $\nu([\Lambda])=(I, \mathbf{r})$ for some integral vector $\mathbf{r}$, and that a homothety class $[\Lambda]$ has type $I$ if any of its elements does. In this case we write $\nu([\Lambda])=I$. We shall denote by $\alpha^{j}$ the $j$-th column of the matrix $\alpha$ and by $D_{i i}$ the $i$-th diagonal entry of $D$. Note that $\alpha$ is only unique up to right-multiplication by an element of

$\Gamma_{I, \mathbf{r}}:=\operatorname{Stab}_{\Gamma}(\Gamma D)=\left\{\left(\begin{array}{c|c|c|c|c}\gamma_{i_{1}} & * & \ldots & * & * \\ \hline p^{r_{i_{1}}} & \gamma_{i_{2}-i_{1}} & \ddots & \vdots & \\ \hline p^{r_{i_{1}}+r_{i_{2} *}} & p^{r_{i_{2} *}} & \ddots & * & \vdots \\ \hline \vdots & \vdots & & \gamma_{i_{l}-i_{l-1}} & * \\ \hline p^{r_{i_{1}}+\cdots+r_{i_{l} *}} & p^{r_{i_{2}}+\cdots+r_{i_{l}} *} & \ldots & p^{r_{i_{l}} *} & \gamma_{n-i_{l}}\end{array}\right)\right\}$

where $\gamma_{\iota}$ stands for a matrix in $\Gamma_{\iota}, *$ for an arbitrary matrix with entries in $\mathbb{Z}_{p}$, and $p^{r} *$ for a matrix with entries in $p^{r} \mathbb{Z}_{p}$ of the appropriate sizes, 
respectively. Thus there is a $1-1$-correspondence between lattice classes $[\Lambda]$ of type $\left(I,\left(r_{i_{1}}, \ldots, r_{i_{l}}\right)\right)$ and cosets $\alpha \Gamma_{I, \mathbf{r}}$. Furthermore

$$
|\{[\Lambda] \mid \nu([\Lambda])=(I, \mathbf{r})\}|=\left|\Gamma: \Gamma_{I, \mathbf{r}}\right|=\mu(\Gamma) / \mu\left(\Gamma_{I, \mathbf{r}}\right)=\left(\begin{array}{c}
n \\
I
\end{array}\right)_{p^{-1}} p^{\sum_{\iota \in I} r_{\iota} \iota(n-\iota)},
$$

where, as usual, $\mu$ denotes the Haar measure on $\Gamma$ normalised so that $\mu(\Gamma)=$ $\left(1-p^{-1}\right) \ldots\left(1-p^{-n}\right)$. As we have noted above it coincides with the additive Haar measure on $\operatorname{Mat}_{n}\left(\mathbb{Z}_{p}\right) \cong \mathbb{Z}_{p}^{n^{2}}$, normalised so that $\mu\left(\operatorname{Mat}_{n}\left(\mathbb{Z}_{p}\right)\right)=1$.

It is now straightforward to check that (24) is equivalent to

$$
\forall i \in[n]: D \mathcal{R}_{(i)}^{<}(\alpha) D \equiv 0 \bmod D_{i i}
$$

where $\mathcal{R}_{(i)}^{<}(\alpha):=\alpha^{-1} \mathcal{R}\left(\alpha^{i}\right)\left(\alpha^{-1}\right)^{t}$. It is easy to verify that condition (27) is equivalent to

$$
\forall i, r, s \in[n]:\left(\mathcal{R}_{(i)}^{<}(\alpha)\right)_{r s} p^{r_{0}+\sum_{s \leq \iota \in I} r_{\iota}+\sum_{r \leq \iota \in I} r_{\iota}+\sum_{i>\iota \in I} r_{\iota}} \equiv 0 \quad \bmod p^{\sum_{\iota \in I} r_{\iota}}
$$

which may in turn be reformulated as

$$
r_{0} \geq \sum_{\iota \in I} r_{\iota}-\underbrace{\min \left\{\sum_{\iota \in I} r_{\iota}, \sum_{s \leq \iota \in I} r_{\iota}+\sum_{r \leq \iota \in I} r_{\iota}+\sum_{i>\iota \in I} r_{\iota}+v_{i r s}(\alpha) \mid(i, r, s) \in[n]^{3}\right\}}_{=: m([\Lambda])},
$$

where $v_{\text {irs }}(\alpha):=\min \left\{v_{p}\left(\left(\mathcal{R}_{(\iota)}^{<}(\alpha)\right)_{\rho \sigma}\right) \mid \iota \leq i, \rho \geq r, \sigma \geq s\right\}$.

Remark 3.1. Whilst it might seem more natural to replace the inequalities in this definition of $v_{\text {irs }}(\alpha)$ by equalities, the present formulation is preferable as it allows us to translate the counting problem into the language developed in Section 2.

Note that the right hand side of (28) depends only on the homothety class of $\Lambda$ and is neatly separated in terms that depend on the type $(I, \mathbf{r})$ of $[\Lambda]$ and terms that depend solely on $\alpha$. The subalgebra $\Lambda_{0}$ is characterised by equality in (28). As we shall see, this formulation of the 'subalgebra condition' (24) therefore enables us to express the $p$-th local zeta function of $L$ in terms of $p$-adic integrals on $\left(p \mathbb{Z}_{p}\right)^{|I|} \times \Gamma, I \subseteq[n-1]$.

By (25) it suffices to compute

$$
A^{<}(s):=\sum_{[\Lambda]}\left|L_{p}: \Lambda_{0}\right|^{-s}=\sum_{I \subseteq[n-1]} \underbrace{\sum_{\nu([\Lambda])=I}\left|L_{p}: \Lambda_{0}\right|^{-s}}_{=: A_{I}^{<}(s)} .
$$

For a fixed $I=\left\{i_{1}, \ldots, i_{l}\right\}_{<} \subseteq[n-1]$, we set

$$
\mathcal{N}_{I, \mathbf{r}, m}^{<}:=|\{[\Lambda] \mid \nu([\Lambda])=(I, \mathbf{r}), m([\Lambda])=m\}|
$$


with $m([\Lambda])$ defined as in $(28)$. Thus

$$
\begin{aligned}
A_{I}^{<}(s) & =\sum_{\mathbf{r}=\left(r_{i_{1}}, \ldots, r_{i_{l}}\right) \in \mathbb{N}^{l}} p^{-s \sum_{\iota \in I} \iota r_{\iota}} \sum_{m \in \mathbb{N}_{0}} \mathcal{N}_{I, \mathbf{r}, m}^{<} p^{-s n\left(\sum_{\iota \in I} r_{\iota}-m\right)} \\
& =\sum_{\mathbf{r} \in \mathbb{N}^{l}} p^{-s \sum_{\iota \in I} r_{\iota}(\iota+n)} \sum_{m \in \mathbb{N}_{0}} \mathcal{N}_{I, \mathbf{r}, m}^{<} p^{s n m}
\end{aligned}
$$

As in the proof of Proposition 2.2 we shall design a $p$-adic integral to describe the generating functions $A_{I}^{<}(s)$. Consider the $p$-adic integral $Z_{I}(\mathbf{s})=$ $Z_{W, \mathbb{Q}_{p}, I}\left(s_{1}, \ldots, s_{n}\right)$ defined as in Section 2 with $k=n, m=n^{2}, W=\Gamma$ and set

$$
\begin{aligned}
\mathbf{f}_{i r s}(\mathbf{y}) & =\left\{\left(\mathcal{R}_{(\iota)}^{<}(\mathbf{y})\right)_{\rho \sigma} \mid \iota \leq i, \rho \geq r, \sigma \geq s\right\},(i, r, s) \in[n]^{3}, \\
\mathbf{g}_{n, I}(\mathbf{x}, \mathbf{y}) & =\left\{\prod_{\iota \in I} x_{\iota}\right\} \cup \bigcup_{(i, r, s) \in[n]^{3}}\left(\prod_{\iota \in I} x_{\iota}^{\delta_{\iota \geq r}+\delta_{\iota \geq s}+\delta_{\iota<i}}\right) \mathbf{f}_{i r s}(\mathbf{y}), \\
\mathbf{g}_{\kappa, I}(\mathbf{x}, \mathbf{y}) & =\left\{\prod_{\iota \in I} x_{\iota}^{\delta_{\iota \kappa}}\right\}, \kappa \in[n-1] .
\end{aligned}
$$

The ideals $\left(\mathbf{f}_{\text {irs }}(\mathbf{y})\right)$ can easily be seen to be $B\left(\mathbb{Q}_{p}\right)$-invariant. Without loss of generality we may assume that none of them equals the zero ideal (otherwise we just omit the respective ideal), and, by omitting at most finitely many primes, we may assume that this also holds $\bmod p$. (Note that we may well have to omit all the $\left(\mathbf{f}_{\text {irs }}(\mathbf{y})\right)$. This happens when the ring structure is trivial.) Thus Theorems 2.2 and 2.3 and their Corollaries apply to a principalisation for the ideal $\mathcal{I}=\prod_{\text {irs }}\left(\mathbf{f}_{\text {irs }}(\mathbf{y})\right)$ with good reduction $\bmod p$. Note that

$$
Z_{I}^{<}(\mathbf{s}):=Z_{I}(\mathbf{s})=Z_{I}\left(\left(s_{\iota}\right)_{\iota \in I}, s_{n}\right)=\sum_{\mathbf{r} \in \mathbb{N}^{l}} p^{-\sum_{\iota \in I} s_{\iota} r_{\iota}} \sum_{m \in \mathbb{N}_{0}} \mu_{I, \mathbf{r}, m}^{<} p^{-s_{n} m},
$$

where

$$
\mu_{I, \mathbf{r}, m}^{<}=\mu\left\{(\mathbf{x}, \mathbf{y}) \in\left(p \mathbb{Z}_{p}\right)^{l} \times \Gamma \mid v_{p}\left(x_{\iota}\right)=r_{\iota}, m(\mathbf{x}, \mathbf{y})=m\right\}
$$

with

$$
\begin{aligned}
& m(\mathbf{x}, \mathbf{y}):= \\
& \min \left\{\sum_{\iota \in I} r_{\iota}, \sum_{s \leq \iota \in I} v_{p}\left(x_{\iota}\right)+\sum_{r \leq \iota \in I} v_{p}\left(x_{\iota}\right)+\sum_{i>\iota \in I} v_{p}\left(x_{\iota}\right)+v_{i r s}(\mathbf{y}) \mid(i, r, s) \in[n]^{3}\right\} .
\end{aligned}
$$

Again we need a lemma to relate the numbers $\mu_{I, \mathbf{r}, m}^{<}$to the data $\mathcal{N}_{I, \mathbf{r}, m}^{<}$we are trying to understand.

\section{Lemma 3.1.}

$$
\mathcal{N}_{I, \mathbf{r}, m}^{<}=\frac{\mu_{I, \mathbf{r}, m}^{<}}{\left(1-p^{-1}\right)^{l} p^{-\sum_{\iota \in I} r_{\iota}} \mu\left(\Gamma_{I, \mathbf{r}}\right)}=\frac{\left(\begin{array}{l}
n \\
I
\end{array}\right)_{p^{-1}}}{\left(1-p^{-1}\right)^{l} \mu(\Gamma)} \mu_{I, \mathbf{r}, m}^{<} p^{\sum_{\iota \in I} r_{\iota}(\iota(n-\iota)+1)} .
$$


Proof. The set

$$
\left\{(\mathbf{x}, \mathbf{y}) \in\left(p \mathbb{Z}_{p}\right)^{l} \times \Gamma \mid v_{p}\left(x_{\iota}\right)=r_{\iota}, m(\mathbf{x}, \mathbf{y})=m\right\}
$$

may be written as a disjoint union of the $\left|\Gamma: \Gamma_{I, \mathbf{r}}\right|$ sets

$$
\left\{(\mathbf{x}, \mathbf{y}) \in\left(p \mathbb{Z}_{p}\right)^{l} \times \gamma \Gamma_{I, \mathbf{r}} \mid v_{p}\left(x_{\iota}\right)=r_{\iota}, m(\mathbf{x}, \mathbf{y})=m\right\}
$$

where $\gamma$ runs through a complete set of coset representatives for $\Gamma / \Gamma_{I, \mathbf{r}}$. The measure of each of these sets is either zero or equal to $\left(1-p^{-1}\right)^{l} p^{-\sum_{\iota \in I} r_{\iota}} \mu\left(\Gamma_{I, \mathbf{r}}\right)$. The latter happens if and only if $\gamma \Gamma_{I, \mathbf{r}}$ corresponds to a lattice of type $(I, \mathbf{r})$ such that $m([\Lambda])=m$. This proves the first equality. The second equality is an immediate consequence of (26).

Lemma 3.1 allows us to express the generating functions (30) in terms of the $p$-adic integrals (32). Indeed,

$$
\begin{aligned}
A_{I}^{<}(s) & =\sum_{\mathbf{r} \in \mathbb{N}^{l}} p^{-s \sum_{\iota \in I} r_{\iota}(\iota+n)} \sum_{m \in \mathbb{N}_{0}} \mathcal{N}_{I, \mathbf{r}, m}^{<} p^{s n m} \\
& =\frac{\left(\begin{array}{l}
n \\
I
\end{array}\right)_{p^{-1}}}{\left(1-p^{-1}\right)^{l} \mu(\Gamma)} \sum_{\mathbf{r}} p^{-\sum_{\iota \in I} r_{\iota}(s(\iota+n)-\iota(n-\iota)-1)} \sum_{m} \mu_{I, \mathbf{r}, m}^{<} p^{s n m} \\
& =\frac{\left(\begin{array}{l}
n \\
I
\end{array}\right)_{p^{-1}}}{\left(1-p^{-1}\right)^{l} \mu(\Gamma)} Z_{I}^{<}\left((s(\iota+n)-\iota(n-\iota)-1)_{\iota \in I},-s n\right) \\
& =\left(\begin{array}{c}
n \\
I
\end{array}\right)_{p^{-1}} \widetilde{Z_{I}^{<}}\left((s(\iota+n)-\iota(n-\iota)-1)_{\iota \in I},-s n\right) .
\end{aligned}
$$

Theorem A follows now from equations (25) and (29), and Corollary 2.3.

3.2. Conjugacy zeta functions of nilpotent groups. In this section we prove Theorem B. As mentioned in the introduction, it suffices to compute

$$
\zeta_{L, p}^{\mathrm{cc}}(s)=\sum_{H \leq L_{p}}\left|L_{p}: H\right|^{-s}\left|L_{p}: \mathcal{N}_{L_{p}}(H)\right|^{-1}
$$

for almost all primes $p$, where $L=L(G), L_{p}=L \otimes \mathbb{Z}_{p}$ and $\mathcal{N}_{L_{p}}(H)=\{l \in$ $\left.L_{p} \mid[H, l] \leq H\right\}$ is the normaliser of $H$ in $L_{p}$.

Remark 3.2. It was pointed out, e.g. in [34, Section 3.8], that, whilst (34) might be used to define local 'conjugacy' zeta functions for arbitrary rings, they might not encode the solutions to any actual counting problem unless $L_{p}=L \otimes \mathbb{Z}_{p}$ for a nilpotent Lie ring $L$. It is for that reason that we formulate Theorem B in terms of $\mathcal{T}$-groups $G$, bearing in mind that its proof is immediately reduced to a computation in the Lie ring $L(G)$ that draws upon neither the nilpotency nor the Lie property of $L(G)$.

Along the lines of the 'first observation' in the proof of Theorem A we use the fact that, for a subring $H$ of $L_{p}$, the normaliser $\mathcal{N}_{L_{p}}(H)$ is an invariant 
of the homothety class $[H]$ of $H$. We may thus write $\mathcal{N}_{L_{p}}([H])$ for $\mathcal{N}_{L_{p}}(H)$. Resuming the notation of the proof of Theorem A we have

$$
L_{p} \geq \mathcal{N}_{L_{p}}([H]) \geq \Lambda_{0} \geq p \Lambda_{0} \geq p^{2} \Lambda_{0} \geq \ldots
$$

$\left(H \in\left\{p^{m} \Lambda_{0} \mid m \in \mathbb{N}_{0}\right\}\right)$. In addition to recording the index $\left|L_{p}: \Lambda_{0}\right|$ as we run over homothety classes $[\Lambda]$ of lattices in $\mathbb{Q}_{p}^{n}$, we now have to control the index $\left|L_{p}: \mathcal{N}_{L_{p}}([\Lambda])\right|$. We shall see that this index, too, might be expressed in terms of congruences involving the type $(I, \mathbf{r})$ and coset $\alpha \Gamma_{I, \mathbf{r}}$ determining $[\Lambda]=\left[\Lambda_{0}\right]$, similar to the congruences (27). To compute their index requires us to control the elementary divisors of certain matrices. A similar problem was considered in Section 2.2; the problem we will have to solve in Section 3.3 is also of this kind (though slightly simpler). We resume the notation from Section 3.1. From (35) we deduce

$$
\zeta_{L, p}^{\mathrm{cc}}(s)=\left(1-p^{-n s}\right)^{-1} \sum_{[\Lambda]}\left|L_{p}: \Lambda_{0}\right|^{-s}\left|L_{p}: \mathcal{N}_{L_{p}}([\Lambda])\right|^{-1} .
$$

Using a fixed basis to identify $L_{p}$ with $\mathbb{Z}_{p}^{n}$ we may express the condition $\mathbf{x} \in \mathcal{N}_{L_{p}}([H])$ in a similar fashion to (24). If $M$ is any matrix whose rows $M_{k}$ encode the coordinates of generators for any element of $[\Lambda]$, a vector $\mathbf{x}=\left(x_{1}, \ldots, x_{n}\right) \in \mathbb{Z}_{p}^{n}$ is in $\mathcal{N}_{L_{p}}([\Lambda])$ if and only if

$$
\forall i \in[n]: \mathbf{x} \sum_{r \in[n]} C_{r} m_{i r} \in\left\langle M_{k} \mid k \in[n]\right\rangle_{\mathbb{Z}_{p}} .
$$

Using the correspondence between lattice classes and pairs $\left((I, \mathbf{r}), \alpha \Gamma_{I, \mathbf{r}}\right)$, condition (36) may be reformulated as

$$
\forall i \in[n]: \mathbf{x} \mathcal{R}_{(i)}^{\mathrm{cc}}(\alpha) D \equiv 0 \bmod D_{i i},
$$

where $\mathcal{R}_{(i)}^{\mathrm{cc}}(\alpha):=\mathcal{R}\left(\alpha^{i}\right)\left(\alpha^{-1}\right)^{t}$ and $D=D(I, \mathbf{r})$. We note that the scalar $p^{r_{0}}$ in $D$ cancels in (37); we may thus assume $r_{0}=0$. Condition (37) is then equivalent to

$$
\forall i \in[n]: \mathbf{x}_{(i)}^{\mathrm{cc}}(\alpha) D p^{\sum_{\iota<i} r_{\iota}} \equiv 0 \quad \bmod p^{\sum_{\iota \in I} r_{\iota}} .
$$

Setting

$$
\begin{aligned}
\mathcal{R}^{\mathrm{cc}}(\alpha) & =\left(\mathcal{R}_{(1)}^{\mathrm{cc}}(\alpha)|\ldots| \mathcal{R}_{(n)}^{\mathrm{cc}}(\alpha)\right), \\
\mathbf{D}^{\mathrm{cc}}(I, \mathbf{r}) & =\operatorname{diag}(\underbrace{D, \ldots, D}_{i_{1}}, \underbrace{p^{r_{i_{1}}} D, \ldots, p^{r_{i_{1}}} D}_{i_{2}-i_{1}}, \ldots, \underbrace{p^{\sum_{\iota \in I} r_{\iota}} D, \ldots, p^{\sum_{\iota \in I} r_{\iota}} D}_{n-i_{l}}),
\end{aligned}
$$

(where 'diag' refers to the diagonal $n^{2} \times n^{2}$-matrix built from $n$ scalar multiples of the diagonal $n \times n$-matrix $D$ ), (38) may in turn be reformulated as

$$
\mathbf{x} \mathcal{R}^{\mathrm{cc}}(\alpha) \mathbf{D}^{\mathrm{cc}}(I, \mathbf{r}) \equiv 0 \quad \bmod p^{\sum_{\iota \in I} r_{\iota}}
$$

To keep track of the index of $\mathcal{N}_{L_{p}}([\Lambda])$, the full sublattice of $L_{p} \cong \mathbb{Z}_{p}^{n}$ of solutions to (39), we introduce an invariant $\nu^{\mathrm{cc}}([\Lambda]) \in \mathbb{N}_{0}^{n}$ as follows. We say that $\nu^{\mathrm{cc}}([\Lambda])=\mathbf{m}=\left(m_{1}, \ldots, m_{n}\right) \in \mathbb{N}_{0}^{n}$ if 
- the matrix $\mathcal{R}^{\mathrm{cc}}(\alpha) \mathbf{D}^{\mathrm{cc}}(I, \mathbf{r})$ has elementary divisor type

$$
\widetilde{\mathbf{m}}=\left(\widetilde{m}_{1}, \ldots, \widetilde{m}_{n}\right)
$$

(i.e. there are matrices $\beta \in \Gamma_{n}, \gamma \in \Gamma_{n^{2}}$ such that $\beta \mathcal{R}^{\mathrm{cc}}(\alpha) \mathbf{D}^{\mathrm{cc}}(I, \mathbf{r}) \gamma=$ $\left(\operatorname{diag}\left(p^{\widetilde{m}_{1}}, \ldots, p^{\widetilde{m}_{n}}\right) \mid 0\right)$ and $\left.\widetilde{m}_{i} \in \mathbb{N}_{0} \cup\{\infty\}, \widetilde{m}_{1} \leq \cdots \leq \widetilde{m}_{n}\right)$ and

- $\mathbf{m}=\left(m_{1}, \ldots, m_{n}\right)$ is defined by $m_{i}=\min \left\{\sum_{\iota \in I} r_{\iota}, \widetilde{m}_{i}\right\}$ for each $i \in[n]$.

The index $\left|L_{p}: \mathcal{N}_{L_{p}}([\Lambda])\right|$ equals

$$
p^{\sum_{i \in[n]}\left(\sum_{\iota \in I} r_{\iota}-m_{i}\right)} .
$$

As in Section 3.1 it is helpful to write

$$
\begin{aligned}
A^{\mathrm{cc}}(s) & :=\sum_{[\Lambda]}\left|L_{p}: \Lambda_{0}\right|^{-s}\left|L_{p}: \mathcal{N}_{L_{p}}([\Lambda])\right|^{-1} \\
& =\sum_{I \subseteq[n-1]} \underbrace{\sum_{\nu([\Lambda])=I}\left|L_{p}: \Lambda_{0}\right|^{-s}\left|L_{p}: \mathcal{N}_{L_{p}}([\Lambda])\right|^{-1}}_{=: A_{I}^{\mathrm{cc}}(s)} .
\end{aligned}
$$

For a fixed $I=\left\{i_{1}, \ldots, i_{l}\right\}_{<} \subseteq[n-1]$, we set

$$
\mathcal{N}_{I, \mathbf{r}, m, \mathbf{m}}^{\mathrm{cc}}:=\left|\left\{[\Lambda] \mid \nu([\Lambda])=(I, \mathbf{r}), m([\Lambda])=m, \nu^{\mathrm{cc}}([\Lambda])=\mathbf{m}\right\}\right|
$$

with $m([\Lambda])$ defined as in $(28)$. We thus have

$$
\begin{aligned}
A_{I}^{\mathrm{cc}}(s) & \\
= & \sum_{\mathbf{r} \in \mathbb{N}^{l}} p^{-s \sum_{\iota \in I} \iota r_{\iota}} \sum_{\substack{m \in \mathbb{N}_{0} \\
\mathbf{m} \in \mathbb{N}_{0}^{n}}} \mathcal{N}_{I, \mathbf{r}, m, \mathbf{m}}^{\mathrm{cc}} p^{-s n\left(\sum_{\iota \in I} r_{\iota}-m\right)-\sum_{i \in[n]}\left(\sum_{\iota \in I} r_{\iota}-m_{i}\right)} \\
= & \sum_{\mathbf{r} \in \mathbb{N}^{l}} p^{\sum_{\iota \in I} r_{\iota}(-s(\iota+n)-n)} \sum_{\substack{m \in \mathbb{N}_{0} \\
\mathbf{m} \in \mathbb{N}_{0}^{n}}} \mathcal{N}_{I, \mathbf{r}, m, \mathbf{m}}^{\mathrm{cc}} p^{s n m+\sum_{i \in[n]} m_{i}} .
\end{aligned}
$$

As in Section 3.1 we shall show that the generating functions $A_{I}^{\mathrm{cc}}(s)$ may be expressed in terms of the $p$-adic integrals to which the results of Section 2 may be applied. In order to control the invariant $\nu^{\mathrm{cc}}([\Lambda])$ we need to parametrise the minors of the matrix $\mathcal{R}^{\mathrm{cc}}(\mathbf{y})$ in a suitable way. This motivates the following combinatorial definitions.

Let $\operatorname{Mat}_{n}(\{0,1\})$ denote the set of $n \times n$-matrices with entries in $\{0,1\}$, and $\mathcal{S}_{j, n}^{\mathrm{cc}}=\left\{S \in \operatorname{Mat}_{n}(\{0,1\}) \mid \sum_{r, s} S_{r s}=j\right\}$. We introduce a partial order on $\mathcal{S}_{j, n}^{\text {cc }}$ by saying that, given $S, T \in \mathcal{S}_{j, n}^{\text {cc }}, T \preceq S$ if,

for all $r \in[n]: \quad \sum_{\rho \leq r} \sum_{\sigma \in[n]} T_{\rho \sigma} \geq \sum_{\rho \leq r} \sum_{\sigma \in[n]} S_{\rho \sigma}$ and,

for all $s \in[n]: \quad \sum_{\sigma>s} \sum_{\rho \in[n]} T_{\rho \sigma} \geq \sum_{\sigma>s} \sum_{\rho \in[n]} S_{\rho \sigma}$.

Pictorially speaking, this amounts to saying that the matrix $T$ may be obtained from the matrix $S$ by moving some of the non-zero entries towards 'north-east'. Given a matrix $S \in \mathcal{S}_{j, n}^{\mathrm{cc}}$ and a $n \times n^{2}$-matrix $M=\left(M_{1}|\ldots| M_{n}\right)$, $M_{i} \in \operatorname{Mat}_{n}\left(\mathbb{Z}_{p}\right)$, a $j \times j$-submatrix of $M$ of column-type $S$ is a submatrix obtained by choosing $j$ rows of $M$ and the $s$-th column of $M_{r}$ if and only if 
$S_{r s}=1$. For $S \in \mathcal{S}_{j, n}^{\mathrm{cc}}$, we set

$\mathbf{f}_{j, S}^{\mathrm{cc}}(\mathbf{y})=\left\{\operatorname{det}(N) \mid N\right.$ a $j \times j$-submatrix of $\mathcal{R}^{\mathrm{cc}}(\mathbf{y})$ of column-type $\left.T \preceq S\right\}$

and define the monomial

$$
M_{S, I}^{\mathrm{cc}}(\mathbf{x})=\prod_{\iota \in I} x_{\iota}^{\sum_{\rho>\iota} \sum_{\sigma=1}^{n} S_{\rho \sigma}+\sum_{\sigma \leq \iota} \sum_{\rho=1}^{n} S_{\rho \sigma}} .
$$

We set

$$
\mathbf{f}_{j, I}^{\mathrm{cc}}(\mathbf{x}, \mathbf{y})=\bigcup_{S \in \mathcal{S}_{j, n}^{\mathrm{cc}}} M_{S, I}^{\mathrm{cc}}(\mathbf{x}) \mathbf{f}_{j, S}^{\mathrm{cc}}(\mathbf{y}) .
$$

Now we define, using the sets of polynomials $\mathbf{g}_{\kappa, I}(\mathbf{x}, \mathbf{y})$ introduced in (31),

$$
\begin{aligned}
& Z_{I}^{\mathrm{cc}}(\mathbf{s}, \widetilde{\mathbf{s}}, \widetilde{\widetilde{\mathbf{s}}})=\int_{\left(p \mathbb{Z}_{p}\right)^{l} \times W} \prod_{\kappa \in[n]}\left\|\mathbf{g}_{\kappa, I}(\mathbf{x}, \mathbf{y})\right\|^{s_{\kappa}} . \\
& \quad \prod_{j \in[n]}\left(\left\|\mathbf{f}_{j, I}^{\mathrm{cc}}(\mathbf{x}, \mathbf{y}) \cup\left(\prod_{\iota \in I} x_{\iota}\right) \mathbf{f}_{j-1, I}^{\mathrm{cc}}(\mathbf{x}, \mathbf{y})\right\|^{\widetilde{s}_{j}}\left\|\mathbf{f}_{j-1, I}^{\mathrm{cc}}(\mathbf{x}, \mathbf{y})\right\|^{\widetilde{s}_{j}}\right)\left|\mathrm{d} \mathbf{x}_{I} \| \mathrm{d} \mathbf{y}\right| .
\end{aligned}
$$

Remark 3.3. Note that $M_{T, I}^{\mathrm{cc}}(\mathbf{x}) \mid M_{S, I}^{\mathrm{cc}}(\mathbf{x})$ if $T \preceq S$. We therefore could have kept definition (41) simpler by replacing ' $T \preceq S$ ' by ' $S$ ', without changing the integral $Z_{I}^{\mathrm{cc}}$. The extra complication ensures that the results from Section 2 are applicable.

We leave it to the reader to verify that, for each $j \in[n]$ and $S \in \mathcal{S}_{j, n}^{\mathrm{cc}}$, the ideal $\left(\mathbf{f}_{j, S}^{c c}(\mathbf{y})\right)$ is $B\left(\mathbb{Q}_{p}\right)$-invariant. Therefore the Theorems 2.2 and 2.3 and their corollaries are applicable to the integral $Z_{I}^{\mathrm{cc}}(\mathbf{s}, \widetilde{\mathbf{s}}, \widetilde{\mathbf{s}})$. Note that $Z_{I}^{\mathrm{cc}}(\mathbf{s}, \widetilde{\mathbf{s}}, \widetilde{\mathbf{s}})=Z_{I}^{\mathrm{cc}}\left(\left(s_{\iota}\right)_{\iota \in I}, s_{n}, \widetilde{\mathbf{s}}, \widetilde{\widetilde{s}}\right)$. We set

$$
\mu_{I, \mathbf{r}, m, \mathbf{m}}^{\mathrm{cc}}=\mu\left\{(\mathbf{x}, \mathbf{y}) \in\left(p \mathbb{Z}_{p}\right)^{l} \times W \mid \nu_{p}\left(\mathbf{x}_{\iota}\right)=r_{\iota}, m(\mathbf{x}, \mathbf{y})=m, \nu^{\mathrm{cc}}(\mathbf{x}, \mathbf{y})=\mathbf{m}\right\} .
$$

Here $\nu^{\mathrm{cc}}(\mathbf{x}, \mathbf{y})=\mathbf{m}=\left(m_{1}, \ldots, m_{n}\right)$ if, for each $i \in[n]$,

$$
m_{i}=\min \left\{\sum_{\iota \in I} v_{p}\left(x_{\iota}\right), \widetilde{m}_{i}\right\},
$$

where $\widetilde{\mathbf{m}}=\left(\widetilde{m}_{1}, \ldots, \widetilde{m}_{n}\right)$ is the elementary divisor type of the matrix

$$
\mathcal{R}^{\mathrm{cc}}(\mathbf{y}) \mathbf{D}^{\mathrm{cc}}\left(I,\left(v_{p}\left(x_{\iota}\right)\right)_{\iota \in I}\right) .
$$

Then

$$
\begin{aligned}
Z_{I}^{\mathrm{cc}}\left(\left(s_{\iota}\right)_{\iota \in I}, s_{n}, \widetilde{\mathbf{s}}\right) & :=Z_{I}^{\mathrm{cc}}\left(\left(s_{\iota}\right)_{\iota \in I}, s_{n}, \widetilde{\mathbf{s}},-\widetilde{\mathbf{s}}\right) \\
& =\sum_{\mathbf{r} \in \mathbb{N}^{l}} p^{-\sum_{\iota \in I} s_{\iota} r_{\iota}} \sum_{\substack{m \in \mathbb{N}_{0} \\
\mathbf{m} \in \mathbb{N}^{n}}} \mu_{I, \mathbf{r}, m, \mathbf{m}}^{\mathrm{cc}} p^{-s_{n} m-\sum_{i \in[n]} \widetilde{s}_{i} m_{i}} .
\end{aligned}
$$

As in (33) we want to relate the numbers $\mu_{I, \mathbf{r}, m, \mathbf{m}}^{\mathrm{cc}}$ with the integers $\mathcal{N}_{I, \mathbf{r}, m, \mathbf{m}}^{\mathrm{cc}}$. 


\section{Lemma 3.2.}

$$
\mathcal{N}_{I, \mathbf{r}, m, \mathbf{m}}^{\mathrm{cc}}=\frac{\left(\begin{array}{l}
n \\
I
\end{array}\right)_{p^{-1}}}{\left(1-p^{-1}\right)^{l} \mu(\Gamma)} \mu_{I, \mathbf{r}, m, \mathbf{m}}^{\mathrm{cc}} p^{\sum_{\iota \in I} r_{\iota}(\iota(n-\iota)+1)} .
$$

Proof. Analogous to the proof of Lemma 3.1.

Thus

$$
\begin{aligned}
& A_{I}^{\mathrm{cc}}(s) \sum_{\mathbf{r} \in \mathbb{N}^{l}} p^{\sum_{\iota \in I} r_{\iota}(-s(\iota+n)-n)} \sum_{\substack{m \in \mathbb{N}_{0}, \mathbf{m} \in \mathbb{N}_{0}^{n}}} \mathcal{N}_{I, \mathbf{r}, m, \mathbf{m}}^{\mathrm{cc}} p^{s n m+\sum_{i \in[n]} m_{i}} \\
&= \frac{\left(\begin{array}{l}
n \\
I
\end{array}\right)_{p^{-1}}}{\left(1-p^{-1}\right)^{l} \mu(\Gamma)} . \\
& \sum_{\mathbf{r} \in \mathbb{N}^{l}} p^{\sum_{\iota} r_{\iota}(-s(\iota+n)+\iota(n-\iota)+1-n)} \sum_{m, \mathbf{m}} \mu_{I, \mathbf{r}, m, \mathbf{m}}^{\mathrm{cc}} p^{s n m+\sum_{i \in[n]} m_{i}} \\
&= \frac{\left(\begin{array}{l}
n \\
I
\end{array}\right)_{p^{-1}}}{\left(1-p^{-1}\right)^{l} \mu(\Gamma)} . \\
& Z_{I}^{\mathrm{cc}}\left((s(\iota+n)-\iota(n-\iota)-1+n)_{\iota \in I},-s n,-1, \ldots,-1\right) \\
&\left(\begin{array}{l}
n \\
I
\end{array}\right)_{p^{-1}} \widetilde{Z_{I}^{\mathrm{cc}}}\left((s(\iota+n)-\iota(n-\iota)-1+n)_{\iota \in I},-s n,-1, \ldots,-1\right) .
\end{aligned}
$$

Theorem B now follows from Corollary 2.3.

3.3. Normal zeta functions of class-2-nilpotent groups. In this section we prove Theorem C. Let $G$ be a $\mathcal{T}_{2}$-group as in the statement of the theorem. Without loss of generality we may assume that $G / Z(G)$ and $Z(G)$ are torsionfree abelian groups of rank $d$ and $d^{\prime}$, respectively (so $n=d+d^{\prime}$ ), and that $G$ admits a presentation

$G=\left\langle g_{1}, \ldots, g_{d}, h_{1}, \ldots, h_{d^{\prime}}\right|\left[g_{i}, g_{j}\right]=\sum_{k \in[d]} \lambda_{i j}^{k} h_{k}, \lambda_{i j}^{k} \in \mathbb{Z}$, all other [,] trivial $\rangle$.

(Note that we used additive notation for expressions in the abelian group $G^{\prime}$.) Thus we obtain a matrix

$$
\mathcal{M}(\mathbf{y}):=\left(L_{i j}(\mathbf{y})\right) \in \operatorname{Mat}_{d}(\mathbb{Z}[\mathbf{y}])
$$

of linear forms $L_{i j}(\mathbf{y}):=\sum_{k \in[d]} \lambda_{i j}^{k} y_{k}$, encoding the commutator structure of $G$. By disregarding at most finitely many further primes we may also assume that $p$ does not divide $\mathcal{M}(\alpha)$ whenever $\alpha \in \mathbb{Z}_{p}^{d^{\prime}} \backslash p \mathbb{Z}_{p}^{d^{\prime}}$.

We begin our argument as in [31, Section 3], albeit with slightly different notation. Note, however, that we do not require the assumption that $Z(G)=$ $G^{\prime}$. By [31, Lemma 1] and its corollary, it suffices to compute a functional equation for the generating function

$$
A^{\triangleleft}(s)=\sum_{I \subseteq\left[d^{\prime}-1\right]} A_{I}^{\triangleleft}(s),
$$


where

$$
A_{I}^{\triangleleft}(s)=\sum_{\nu([\Lambda])=I}\left|Z\left(L_{p}\right): \Lambda\right|^{d-s}\left|L_{p}: X(\Lambda)\right|^{-s} .
$$

Here the sum ranges over homothety classes of maximal lattices $\Lambda$ of type ${ }^{2} I$ in the centre $Z\left(L_{p}\right)$ of the $\mathbb{Z}_{p}$-algebra

$$
L_{p}:=(G / Z(G) \oplus Z(G)) \otimes \mathbb{Z}_{p},
$$

and $X(\Lambda) / \Lambda=Z\left(L_{p} / \Lambda\right)$. We identify $Z\left(L_{p}\right)$ with $\mathbb{Z}_{p}^{d^{\prime}}$ using the basis $\left\{h_{1}, \ldots, h_{d^{\prime}}\right\}$. The index $\left|L_{p}: X(\Lambda)\right|$ is the index in $L_{p} / Z\left(L_{p}\right) \cong \mathbb{Z}_{p}^{d}$ of a system of linear congruences which we now describe (cf. $[30, \S 2.2])$. Let $[\Lambda]$ be of type $(I, \mathbf{r})$, $I=\left\{i_{1}, \ldots, i_{l}\right\}_{<} \subseteq\left[d^{\prime}-1\right], \mathbf{r}=\left(r_{i_{1}}, \ldots, r_{i_{l}}\right) \in \mathbb{N}^{l}$, corresponding to the coset $\alpha \Gamma_{I, \mathbf{r}} \in \Gamma_{d^{\prime}} / \Gamma_{I, \mathbf{r}}$ as in the proof of Theorem A. We set

$$
\begin{aligned}
\mathcal{M}^{\triangleleft}(\alpha) & =\left(\mathcal{M}\left(\alpha^{1}\right)|\ldots| \mathcal{M}\left(\alpha^{d^{\prime}}\right)\right), \\
\mathbf{D}^{\triangleleft}(I, \mathbf{r}) & =\operatorname{diag}(\underbrace{1, \ldots, 1}_{d i_{1}}, \underbrace{p^{r_{i_{1}}}, \ldots, p^{r_{i_{1}}}}_{d\left(i_{2}-i_{1}\right)}, \ldots, \underbrace{p^{\sum_{\iota \in I} r_{\iota}}, \ldots, p^{\sum_{\iota \in I} r_{\iota}}}_{d\left(d^{\prime}-i_{l}\right)}) .
\end{aligned}
$$

The system of linear congruences under consideration is

$$
\mathbf{x} \mathcal{M}^{\triangleleft}(\alpha) \mathbf{D}^{\triangleleft}(I, \mathbf{r}) \equiv 0 \quad \bmod p^{\sum_{\iota \in I} r_{\iota}} .
$$

The solutions to this system form a full lattice in $L_{p} / Z\left(L_{p}\right)$. To keep track of its index we define the invariant $\nu^{\prime}([\Lambda]) \in \mathbb{N}_{0}^{d}$ as follows. We say that $\nu^{\prime}([\Lambda])=\mathbf{m}=\left(m_{1}, \ldots, m_{d}\right)$ if

- the matrix $\mathcal{M}^{\triangleleft}(\alpha) \mathbf{D}^{\triangleleft}(I, \mathbf{r})$ has elementary divisor type

$$
\widetilde{\mathbf{m}}=\left(\widetilde{m}_{1}, \ldots, \widetilde{m}_{d}\right)
$$

and

- $\mathbf{m}=\left(m_{1}, \ldots, m_{n}\right)$ is defined by $m_{i}=\min \left\{\sum_{\iota \in I} r_{\iota}, \tilde{m}_{i}\right\}$ for each $i \in[n]$.

The index of the system (45) equals

$$
p^{\sum_{j \in[d]}\left(\sum_{\iota \in I} r_{\iota}-m_{j}\right)} .
$$

By defining

$$
\mathcal{N}_{I, \mathbf{r}, \mathbf{m}}^{\triangleleft}:=\left|\left\{[\Lambda] \mid \nu([\Lambda])=(I, \mathbf{r}), \nu^{\prime}([\Lambda])=\mathbf{m}\right\}\right|
$$

(note that $\mathcal{N}_{I, \mathbf{r}, \mathbf{m}}^{\triangleleft} \neq 0$ implies $m_{1}=0$, as $p \not \mathcal{M}^{\triangleleft}(\alpha)$ ) we obtain a formula for $A_{I}^{\triangleleft}(s)$ that is analogous to $(30)$ :

$$
\begin{aligned}
A_{I}^{\triangleleft}(s) & =\sum_{\mathbf{r} \in \mathbb{N}^{l}} p^{(d-s) \sum_{\iota \in I} r_{\iota} \iota} \sum_{\mathbf{m} \in \mathbb{N}_{0}^{d}} \mathcal{N}_{I, \mathbf{r}, \mathbf{m}}^{\triangleleft} p^{-s \sum_{j \in[d]}\left(\sum_{\iota \in I} r_{\iota}-m_{j}\right)} \\
& =\sum_{\mathbf{r}} p^{\sum_{\iota \in I} r_{\iota}(-s(d+\iota)+\iota d)} \sum_{\mathbf{m}} \mathcal{N}_{I, \mathbf{r}, \mathbf{m}}^{\triangleleft} p^{s \sum_{j \in[d]} m_{j}} .
\end{aligned}
$$

\footnotetext{
${ }^{2}$ Note that the definition of a lattice's type given in [31] differs from the one in the current paper in so far as $I$ is replaced by $d^{\prime}-I=\left\{d^{\prime}-i \mid i \in I\right\}$.
} 
As in Section 3.1 we shall show that the generating function $A_{I}^{\triangleleft}(s)$ may be expressed in terms of a $p$-adic integral to which the results of Section 2 can be applied. We shall need some more notation.

Given the $d \times d d^{\prime}$-matrix $M=\left(M_{1}|\ldots| M_{d^{\prime}}\right), M_{i} \in \operatorname{Mat}_{d}\left(\mathbb{Z}_{p}\right)$, a $j \times j$ submatrix of $M$ of column-type $S=\left(\sigma_{1}, \ldots, \sigma_{d^{\prime}}\right) \in \mathbb{N}_{0}^{d^{\prime}}, \sum_{i \in\left[d^{\prime}\right]} \sigma_{i}=j$, is a submatrix obtained by choosing $j$ rows of $M$ and $\sigma_{i}$ columns in the 'block' $M_{i}$ for each $i \in\left[d^{\prime}\right]$. We denote by $\mathcal{S}_{j, d^{\prime}}^{\triangleleft}=\left\{\left(\sigma_{1}, \ldots, \sigma_{d^{\prime}}\right) \mid \sum_{i \in\left[d^{\prime}\right]} \sigma_{i}=j\right\}$ the set of possible such column-types. Given $S=\left(\sigma_{i}\right), T=\left(\tau_{i}\right) \in \mathcal{S}_{j, d^{\prime}}^{\triangleleft}$, we write $T \preceq S$ if, for all $i \in\left[d^{\prime}\right], \sum_{\iota \leq i} \tau_{\iota} \geq \sum_{\iota \leq i} \sigma_{\iota}$. For $S \in \mathcal{S}_{j, d^{\prime}}^{\triangleleft}$, we set

$$
\mathbf{f}_{j, S}^{\triangleleft}(\mathbf{y})=\{\operatorname{det}(N) \mid N \text { a } j \times j \text {-submatrix of } \mathcal{M}(\mathbf{y}) \text { of column-type } T \preceq S\} \text {. }
$$

We define the monomial $M_{S, I}^{\triangleleft}(\mathbf{x})=\prod_{\iota \in I} x_{\iota}^{\sum_{\iota<\kappa \in\left[d^{\prime}\right]} \sigma_{\kappa}}$ and set

$$
\mathbf{f}_{j, I}^{\triangleleft}(\mathbf{x}, \mathbf{y})=\bigcup_{S \in \mathcal{S}_{j, d^{\prime}}^{\triangleleft}} M_{S, I}^{\triangleleft}(\mathbf{x}) \mathbf{f}_{j, S}^{\triangleleft}(\mathbf{y}) .
$$

We are now ready to define

$$
\begin{aligned}
& Z_{I}^{\triangleleft}(\mathbf{t}, \mathbf{s}, \widetilde{\mathbf{s}})=Z_{I}^{\triangleleft}\left(\left(t_{\iota}\right)_{\iota \in I}, s_{2}, \ldots, s_{d}, \widetilde{s}_{2}, \ldots, \widetilde{s}_{d}\right)=\int_{\left(p \mathbb{Z}_{p}\right)^{l} \times W} \prod_{\iota \in I}\left|x_{\iota}\right|^{t_{\iota}} . \\
& \prod_{j \in[2, d]}\left(\left\|\mathbf{f}_{j, I}^{\triangleleft}(\mathbf{x}, \mathbf{y}) \cup\left(\prod_{\iota \in I} x_{\iota}\right) \mathbf{f}_{j-1, I}^{\triangleleft}(\mathbf{x}, \mathbf{y})\right\|^{s_{j}}\left\|\mathbf{f}_{j-1, I}^{\triangleleft}(\mathbf{x}, \mathbf{y})\right\|^{\widetilde{s}_{j}}\right)\left|\mathrm{d} \mathbf{x}_{I} \| \mathrm{d} \mathbf{y}\right| .
\end{aligned}
$$

Remark 3.4. Note that $M_{T, I}^{\triangleleft}(\mathbf{x}) \mid M_{S, I}^{\triangleleft}(\mathbf{x})$ if $T \preceq S$. We could have kept definition (47) simpler by replacing ' $T \preceq S$ ' by ' $S$ '. The extra complication ensures that the results from Section 2 are applicable. Note also that we are not losing anything by omitting the factor for $j=1$, as $\left\|\mathbf{f}_{0, I}^{\triangleleft}(\mathbf{x}, \mathbf{y})\right\|=$ $\left\|\mathbf{f}_{1, I}^{\triangleleft}(\mathbf{x}, \mathbf{y})\right\|=1$ for all $\mathbf{x} \in\left(p \mathbb{Z}_{p}\right)^{l}, \mathbf{y} \in W$.

We leave it to the reader to verify that, for each $j \in[d]$ and $S \in \mathcal{S}_{j, d^{\prime}}^{\triangleleft}$, the ideal $\left(\mathbf{f}_{j, S}^{\triangleleft}(\mathbf{y})\right)$ is $B\left(\mathbb{Q}_{p}\right)$-invariant. Therefore Theorems $2.2,2.3$ and their corollaries are applicable. We set

$$
\mu_{I, \mathbf{r}, \mathbf{m}}^{\triangleleft}=\mu\left\{(\mathbf{x}, \mathbf{y}) \in\left(p \mathbb{Z}_{p}\right)^{l} \times W \mid v_{p}\left(x_{\iota}\right)=r_{\iota}, \nu^{\prime}(\mathbf{x}, \mathbf{y})=\mathbf{m}\right\},
$$

where $\nu^{\prime}(\mathbf{x}, \mathbf{y})=\mathbf{m}=\left(m_{1}, \ldots, m_{d}\right) \in \mathbb{N}_{0}^{d}$ if $\mathbf{m}=\min \left\{\sum_{\iota \in I} r_{\iota}, \widetilde{\mathbf{m}}\right\}$ where $\widetilde{\mathbf{m}}$ is the elementary divisor type of the matrix $\mathcal{M}^{\triangleleft}(\mathbf{y}) \mathbf{D}^{\triangleleft}\left(I,\left(v_{p}\left(x_{\iota}\right)\right)_{\iota \in I}\right)$. Note that $\mu_{I, \mathbf{r}, \mathbf{m}}^{\triangleleft} \neq 0$ implies $m_{1}=0$. Then

$$
Z_{I}^{\triangleleft}\left(\left(t_{\iota}\right)_{\iota \in I}, \mathbf{s}\right):=Z_{I}^{\triangleleft}\left(\left(t_{\iota}\right)_{\iota \in I}, \mathbf{s},-\mathbf{s}\right)=\sum_{\mathbf{r} \in \mathbb{N}^{l}} p^{-\sum_{\iota \in I} t_{\iota} r_{\iota}} \sum_{\mathbf{m} \in \mathbb{N}_{0}^{d}} \mu_{I, \mathbf{r}, \mathbf{m}}^{\triangleleft} p^{-\sum_{j \in[2, d]} s_{j} m_{j}} .
$$

\section{Lemma 3.3.}

$$
\mathcal{N}_{I, \mathbf{r}, \mathbf{m}}^{\triangleleft}=\frac{\left(\begin{array}{c}
d^{\prime} \\
I
\end{array}\right)_{p^{-1}}}{\left(1-p^{-1}\right)^{l} \mu(\Gamma)} \mu_{I, \mathbf{r}, \mathbf{m}}^{\triangleleft} p^{\sum_{\iota \in I} r_{\iota}\left(\iota\left(d^{\prime}-\iota\right)+1\right)} .
$$


Proof. Analogous to the proof of Lemma 3.1.

Thus

$$
\begin{aligned}
A_{I}^{\triangleleft}(s) & =\sum_{\mathbf{r}} p^{\sum_{\iota \in I} r_{\iota}(-s(d+\iota)+\iota d)} \sum_{\mathbf{m}} \mathcal{N}_{I, \mathbf{r}, \mathbf{m}}^{\triangleleft} p^{s \sum_{j \in[d]} m_{j}} \\
& =\frac{\left(\begin{array}{l}
d^{\prime} \\
I
\end{array}\right)_{p^{-1}}}{\left(1-p^{-1}\right)^{l} \mu(\Gamma)} \cdot \\
& \sum_{\mathbf{r}} p^{\sum_{\iota \in I} r_{\iota}\left(-s(d+\iota)+\iota\left(d^{\prime}+d-\iota\right)+1\right)} \sum_{\mathbf{m}} \mu_{I, \mathbf{r}, \mathbf{m}}^{\triangleleft}\left(p^{-s}\right)^{-\sum_{j \in[d]} m_{j}} \\
= & \frac{\left(\begin{array}{c}
d^{\prime} \\
I
\end{array}\right)_{p^{-1}}}{\left(1-p^{-1}\right)^{l} \mu(\Gamma)} Z_{I}\left(\left(s(d+\iota)-\iota\left(d+d^{\prime}-\iota\right)-1\right)_{\iota \in I},-s, \ldots,-s\right) \\
= & \left(\begin{array}{c}
d^{\prime} \\
I
\end{array}\right)_{p^{-1}} \widetilde{Z_{I}}\left(\left(s(d+\iota)-\iota\left(d+d^{\prime}-\iota\right)-1\right)_{\iota \in I},-s, \ldots,-s\right) .
\end{aligned}
$$

Theorem C now follows from Corollary 2.3.

3.4. Representation zeta functions of $\mathcal{T}$-groups. In this section we recall some of Howe's work [16] on irreducible representations of $\mathcal{T}$-groups and co-adjoint orbits and prove Theorem D.

If $G$ is a group and $H \leq G$ is a subgroup we say that $H$ is saturated if $g^{n} \in H$ implies $g \in H$ for all $g \in G$. Denote by $H_{s}$ the smallest saturated subgroup of $G$ containing $H$ (this is called the isolator of $H$ in [26, Chapter 8, Section A]). Clearly, if $H \triangleleft G, H=H_{s}$ if and only if $G / H$ is torsion-free.

Now let $G$ be a $\mathcal{T}$-group. Recall (cf., for example, [26, Chapter 6]) that, by the Malcev correspondence, there is a Lie algebra $\mathcal{L}_{G}(\mathbb{Q})$ over $\mathbb{Q}$, of dimension equal to $h(G)$, the Hirsch length of $G$, and an injective mapping $\log : G \rightarrow \mathcal{L}_{G}(\mathbb{Q})$, such that $\operatorname{span}_{\mathbb{Q}}(\log (G))=\mathcal{L}_{G}(\mathbb{Q})$. In general $L:=\log (G)$ will fail to be a Lie subring (or even just a lattice). However, by replacing $G$ by a subgroup of finite index, if necessary, we may assume it is a Lie subring ([14], Section 4) and even that $[L, L] \subseteq c ! L$, where $c$ is the nilpotency class of $L$ (or $G$ ), i.e. that $L$ (and $G$ ) are elementarily exponentiable (e.e.) in Howe's nomenclature. As we are looking to study $\zeta_{G, p}^{\text {irr }}(s)$ for all but finitely many primes, there is no harm in descending to a subgroup $H$ of finite index in $G$. Indeed, for all $p$ and all $n$, there is a $1-1$ - correspondence between twist isoclasses of irreducible characters of degree $p^{n}$ and $p$-admissible twist isoclasses of degree $p^{n}$ of $\widehat{G}_{p}$, the pro-p-completion of $G$ ([17], Lemma 8.5). However, $\widehat{G}_{p} \cong \widehat{H}_{p}$ if $p \nmid|G: H|$.

For a $\mathcal{T}$-group $G$, denote by $\left(G^{(i)}\right)$ the group's lower central series, defined by $G^{(1)}=G, G^{(i+1)}=\left[G^{(i)}, G\right], i \geq 2$. We say that $G \neq\{1\}$ has nilpotency class $c$ (or is step-c-nilpotent) if $G^{(c)} \neq\{1\}$ but $G^{(c+1)}=\{1\}$. Similarly, we denote by $\left(L^{(i)}\right)$ the lower central series of the Lie algebra $L$, defined by $L^{(1)}=L, L^{(i+1)}=\left[L^{(i)}, L\right], i \geq 2$, and we hope that there will be no confusion between group commutators and Lie brackets. It is well-known 
that $\log$ induces a bijection between $L^{(i)}$ and $G^{(i)}, i \in[c]$. We write $G^{\prime}$ for $G^{(2)}$, and $L^{\prime}$ for $L^{(2)}$. By $Z(L)$ we denote the centre of $L$.

For a sublattice $M \subseteq L=\log (G)$, denote its dual $\operatorname{Hom}\left(M, \mathbb{C}^{*}\right)$ by $\widehat{M}$ and by $r_{M}: \widehat{L} \rightarrow \widehat{M},\left.\psi \mapsto \psi\right|_{M}$ the restriction to $M$. We say that $\psi \in \widehat{L}$ is rational on $M$ if $r_{M}(\psi)$ is a torsion element. Clearly $\psi$ is rational on $M$ if and only if $\psi$ is rational on $M_{s}$ if and only if $\psi(n M)=1$ for some $n \in \mathbb{N}$.

Recall that the adjoint action $\operatorname{Ad}$ of $G$ on $L$ is given by

$$
l \mapsto l+[\log \gamma, l]+(\text { higher terms) }
$$

where we omitted Lie terms of degree $\geq 3$. These may be computed in terms of the Baker-Campbell-Hausdorff-formula. This element $F(X, Y) \in \widehat{\mathcal{L}}_{\{X, Y\}}$, the completion of the free Lie algebra on the symbols $X$ and $Y$, provides the solution to

$$
\exp (F(x, y))=\exp (x) \exp (y) .
$$

The Baker-Campbell-Hausdorff-formula allows us therefore to recover the group structure on the Lie algebra (cf. [20, Chapter 9] and [27, V.3.4 and IV.7]). By (50), the co-adjoint action $\mathrm{Ad}^{*}$ of $G$ on $\widehat{L}$ is thus given by

$$
\operatorname{Ad}^{*} \gamma(\psi)(l)=\psi(l) \psi([\log \gamma, l]) \psi(\text { higher terms). }
$$

Given $\psi \in \widehat{L}$ we define a bi-additive, anti-symmetric form $B_{\psi}: L \times L \rightarrow \mathbb{C}^{*}$ by setting $B_{\psi}\left(\left(l_{1}, l_{2}\right)\right)=\psi\left(\left[l_{1}, l_{2}\right]\right)$. We say that a subalgebra $P \leq L$ polarises $B_{\psi}$ if $\left.B_{\psi}\right|_{P \times P}=1$ and it is a maximal additive subgroup with respect to this property. Note that any such additive subgroup contains the $\operatorname{radical} \operatorname{Rad}_{\psi}=$ $\{l \in L \mid \psi([l, L])=1\}$ of $B_{\psi}$.

Lemma [16, Lemmata 1-4] Given $\psi \in \widehat{L}$, the isotropy subgroup $G_{\psi}$ of $\psi$ under $\mathrm{Ad}^{*} G$ is an e.e. subgroup of $G$ and $\log G_{\psi}=\operatorname{Rad}_{\psi}$. If $\psi \in \widehat{L}$ is $\mathrm{Ad}^{*} G$-invariant, then $\psi^{2}$ defines a one-dimensional character on $G$. The orbit of $\psi \in \widehat{L}$ under the co-adjoint action $\mathrm{Ad}^{*}$ of $G$ is finite if and only if $\psi$ is rational on $\log G_{s}^{\prime}$. If $\psi \in \widehat{L}$ is rational on $\log G_{s}^{\prime}$ then there are e.e. polarising subalgebras $P$ for $B_{\psi}$. They have finite index in $L$ satisfying $\mid L$ : $P|=| P: \operatorname{Rad}_{\psi} \mid$.

Theorem [16, Theorem 1(a)] Let $G$ be an e.e. $\mathcal{T}$-group and set $L=\log G$, $L_{s}^{\prime}=\log G_{s}^{\prime}$. Let $\Omega$ be a finite $\operatorname{Ad}^{*} G$-orbit in $\widehat{L}$, and $\psi \in \Omega$. Let $N$ be the period of $\psi$ and assume $N$ to be odd. A finite-dimensional irreducible representation $U_{\Omega}$ may be associated to $\Omega$ in the following manner: Let $P$ be an e.e. polarising subalgebra for $B_{\psi}$, set $\Pi=\exp P$ and $\tilde{\psi}:=\left.\psi\right|_{\Pi}$, a linear character on $\Pi$. Put $U_{\Omega}:=\operatorname{Ind}_{\Pi}^{G} \tilde{\psi}$. Then the dimension of $U_{\Omega}$ is $|\Omega|^{1 / 2}$, and the character of $U_{\Omega}$ is $\xi_{\Omega}=\frac{1}{|\Omega|^{1 / 2}} \sum_{\phi \in \Omega} \phi$. All representations of the form $\chi \otimes V, \chi \in \widehat{G / G_{s}^{\prime}}, V$ defined modulo $N G:=\exp (N \cdot L), N$ odd, are realised in this manner. 
Corollary 3.1. For almost all primes $p$,

$$
\zeta_{G, p}^{\mathrm{irr}}(s)=\sum_{\begin{array}{c}
\psi \in \widehat{L_{s}^{\prime}} \\
\psi \text { rational of } \\
p \text {-power period }
\end{array}}\left|L: \operatorname{Rad}_{\psi}\right|^{-s / 2}\left|L: L_{\psi, 2}\right|^{-1},
$$

where $L_{\psi, 2}=\left\{l \in L \mid \psi\left(\left[l, L_{s}^{\prime}\right]\right)=1\right\}$.

Proof. For primes $p$ not dividing $2\left|G_{s}^{\prime}: G^{\prime}\right|$, Howe's theorem yields that we count every $p$-power degree twist-isoclass at least once when we sum $\mid G$ : $\left.G_{\psi}\right|^{-s / 2}$ over the rational characters $\psi$ of $L_{s}^{\prime}$ of $p$-power period. By further excluding finitely many primes, we may assume that $\left|G: G_{\psi}\right|=\left|L: \operatorname{Rad}_{\psi}\right|$ (cf. [14, Lemma 4.8], in which it is established that log induces an indexpreserving correspondence between $p$-power index subgroups of $G$ and $p$ power index subalgebras of $L$ away from a finite number of primes). Hereby we overcount every orbit by exactly the index $\left|G: G_{\psi, 2}\right|$, where $G_{\psi, 2}=\{\gamma \in$ $\left.G \mid \psi\left(\left[\log \gamma, L_{s}^{\prime}\right]\right)=1\right\}$ is the stabiliser of $\psi$ under the coadjoint action of $G$ on the restriction of characters to $L_{s}^{\prime}$. (Note that this index is always equal to 1 if $G$ is class-2-nilpotent.) Again at the cost of at most finitely many primes we may assume $\left|G: G_{\psi, 2}\right|=\left|L: L_{\psi, 2}\right|$.

Howe's parametrisation of irreducible representations allows us to prove

Proposition 3.1. Let $G$ be a $\mathcal{T}$-group. Then there are matrices $\mathcal{S} \subset \mathcal{R}$ of homogeneous $\mathbb{Q}$-linear forms such that, for almost all primes $p$,

$$
\zeta_{G, p}^{\mathrm{irr}}(s)=P_{\mathcal{R}, \mathcal{S}, \mathbb{Q}_{p}}(s / 2, \ldots, s / 2 ; 1, \ldots, 1),
$$

where $P_{\mathcal{R}, \mathcal{S}, \mathbb{Q}_{p}}$ is the generating function defined in Section 2.2. Proposition 2.2 is applicable for $n=h\left(G^{\prime}\right)$.

Theorem D clearly follows from this.

Proof. We aim to express both factors in the summands of (51) in terms of elementary divisors of matrices of linear forms. Recall that $L$ is additively isomorphic to $\mathbb{Z}^{h}$, where $h$ is the Hirsch length of $G$. Without loss of generality we may assume that $L^{\prime}$ is saturated in $L$, and that $L^{\prime} \cap Z(L)$ is saturated in $Z(L)$ (otherwise we disregard finitely many primes). We fix a $\mathbb{Z}$-basis

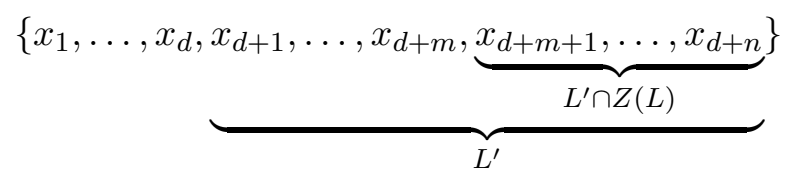

of $L$ such that

$$
\begin{gathered}
\left\{x_{d+1}, \ldots, x_{d+n}\right\} \text { is a } \mathbb{Z} \text {-basis for } L^{\prime} \\
\left\{x_{d+m+1}, \ldots, x_{d+n}\right\} \text { is a } \mathbb{Z} \text {-basis for } L^{\prime} \cap Z(L) .
\end{gathered}
$$

The Lie bracket induces an anti-symmetric, bi-additive mapping

$$
\begin{aligned}
\beta: L /\left(L^{\prime} \cap Z(L)\right) \times L /\left(L^{\prime} \cap Z(L)\right) & \rightarrow L^{\prime} \\
\left(l_{1}\left(L^{\prime} \cap Z(L)\right), l_{2}\left(L^{\prime} \cap Z(L)\right)\right) & \mapsto\left[l_{1}, l_{2}\right]
\end{aligned}
$$


We may express this map in terms of our chosen basis as follows. For $1 \leq$ $i, j \leq d+m$, let

$$
\left[x_{i}, x_{j}\right]=\sum_{k \in[n]} \lambda_{i j k} x_{d+k}, \lambda_{i j k} \in \mathbb{Q}
$$

Let $\mathcal{R}=\left(\mathcal{R}_{i j}\right)$ denote the $(d+m) \times(d+m)$-matrix of linear forms

$$
\mathcal{R}_{i j}(\mathbf{Y})=\sum_{k \in[n]} \lambda_{i j k} Y_{k} \in \mathbb{Q}\left[Y_{1}, \ldots, Y_{n}\right]
$$

By $\mathcal{S}$ we denote the submatrix of $\mathcal{R}$ consisting of the last $m$ columns of $\mathcal{R}$. By further disregarding finitely many primes if necessary we may assume that $p$ divides none of the denominators of the $\lambda_{i j k}$ (so that we may reduce $\bmod p$ ), and that, given $\mathbf{y} \in \mathbb{Z}_{p}^{n}, \overline{\mathcal{R}(\mathbf{y})}$ is not zero unless $\overline{\mathbf{y}}=0 \in \mathbb{F}_{p}^{n}$. We denote by $C_{i}, i \in[d+m]$, the matrices of the additive maps $L /\left(L^{\prime} \cap Z(L)\right) \rightarrow$ $L^{\prime}, x\left(L^{\prime} \cap Z(L)\right) \mapsto\left[x, x_{i}\right]$ with respect to these bases. For a given non-negative integer $N \in \mathbb{N}_{0}$ we identify the set

$$
\Psi_{p^{N}}:=\left\{\psi \in \widehat{L^{\prime}} \mid \text { the period of } \psi \text { equals } p^{N}\right\}
$$

with $\left(\mathbb{Z}^{n} / p^{N} \mathbb{Z}^{n}\right)^{\times}:=\left(\mathbb{Z} / p^{N}\right)^{n} \backslash\left(p \mathbb{Z} / p^{N}\right)^{n}$ by sending $\ell=\left(l_{1}, \ldots, l_{n}\right) \in$ $\left(\mathbb{Z}^{n} / p^{N} \mathbb{Z}^{n}\right)^{\times}$to

$$
\psi\left(b_{1}, \ldots, b_{n}\right)=\exp \left(\frac{2 \pi i \sum_{i \in[n]} l_{i} b_{i}}{p^{N}}\right), \mathbf{b}=\left(b_{1}, \ldots, b_{n}\right) \in \widehat{L}^{\prime}
$$

With these identifications we obtain

$$
\begin{aligned}
\mathbf{k}=\left(k_{1}, \ldots, k_{d+m}\right) \in \operatorname{Rad}_{\psi} / Z(L) & \Leftrightarrow \forall \gamma \in G: \psi\left(\left[\sum k_{i} x_{i}, \log \gamma\right]\right)=1 \\
& \Leftrightarrow \forall j \in[d+m]: \mathbf{k} C_{j} \ell^{t} \equiv 0 \bmod p^{N} \\
& \Leftrightarrow \mathbf{k} \mathcal{R}(\ell) \equiv 0 \bmod p^{N}
\end{aligned}
$$

and

$$
\begin{aligned}
\mathbf{k}=\left(k_{1}, \ldots, k_{d+m}\right) \in L_{\psi, 2} / Z(L) & \Leftrightarrow \forall \gamma \in G^{\prime}: \psi\left(\left[\sum k_{i} x_{i}, \log \gamma\right]\right)=1 \\
& \Leftrightarrow \forall j \in[d+1, d+m]: \mathbf{k} C_{j} \ell^{t} \equiv 0 \bmod p^{N} \\
& \Leftrightarrow \mathbf{k} \mathcal{S}(\ell) \equiv 0 \bmod p^{N} .
\end{aligned}
$$

In order to use the congruence conditions (52) and (53) for an effective computation of $\zeta_{G, p}^{\text {irr }}(s)$, we need to enumerate the (p-parts of) the elementary divisors of the matrices $\mathcal{R}(\ell)$ and $\mathcal{S}(\ell)$ as $\ell$ runs through the sets $\Psi_{p^{N}}, N \in \mathbb{N}$. 
By Corollary 3.1 we may write

$$
\begin{aligned}
\zeta_{G, p}^{\mathrm{irr}}(s)= & \sum_{\substack{N \in \mathbb{N}_{0} \\
\psi \in \Psi_{p^{N}}}}\left|L: \operatorname{Rad}_{\psi}\right|^{-s / 2}\left|L: L_{\psi, 2}\right|^{-1} \\
= & \sum_{\substack{N \in \mathbb{N}_{0} \\
\mathbf{m} \in \mathbb{N}_{0}^{d+m}, \mathbf{n} \in \mathbb{N}_{0}^{m}}} \mathcal{N}_{N, \mathbf{m}, \mathbf{n}} q^{-\sum_{i \in[d+m]}\left(N-m_{i}\right)^{s / 2}-\sum_{j \in[m]}\left(N-n_{j}\right)} \\
= & P_{\mathcal{R}, \mathcal{S}, \mathbb{Q}_{p}}(s / 2, \ldots, s / 2 ; 1, \ldots, 1) .
\end{aligned}
$$

Acknowledgements. I started work on this paper when I was a guest at the Max-Planck-Institut für Mathematik in Bonn, Germany. I continued it as a guest at the Chaire de Mathématiques Algébriques Discrètes at the EPF Lausanne, Switzerland. I gratefully acknowledge both institution's support and hospitality. I am particularly obliged to Laurent Bartholdi, my host in Lausanne, and to the Fond National Suisse, which jointly funded my stay there. Thanks are due to Benjamin Klopsch for frequent inspiring discussions. I wish to thank Benjamin Martin for making Hrushovski's and his preprint [17] available to me, and to Willem Veys for alerting me to [29]. Mark Berman's and Ulrich Voll's comments on previous versions helped a great deal to improve the presentation of this paper. I am particularly grateful to Mark Berman for his help with proof-reading.

\section{REFERENCES}

[1] M.N. Berman, Uniformity and functional equations for local zeta functions of $\mathbb{Q}$-split algebraic groups, preprint, 2006.

[2] C.W. Curtis and I. Reiner, Methods of representation theory, with applications to finite groups and orders, vol. 1, John Wiley \& Sons, 1981.

[3] J. Denef, On the degree of Igusa's local zeta function, Amer. J. Math. 109 (1987), no. 6, 991-1008.

[4] Report on Igusa's local zeta function, Séminaire Bourbaki 43 (1990-91), no. 201-203, 359-386.

[5] J. Denef and D. Meuser, A functional equation of Igusa's local zeta function, Amer. J. Math. 113 (1991), no. 6, 1135-1152.

[6] M.P.F. du Sautoy, A nilpotent group and its elliptic curve: non-uniformity of local zeta functions of groups, Israel J. Math. 126 (2001), 269-288.

[7] _ Counting subgroups in nilpotent groups and points on elliptic curves, J. Reine Angew. Math. 549 (2002), 1-21.

[8] M.P.F. du Sautoy and F.J. Grunewald, Analytic properties of zeta functions and subgroup growth, Ann. of Math. 152 (2000), 793-833.

[9] Z Z Z Z L functions of groups and rings, Proceedings of the International Congress of Mathematicians, Madrid, August 22-30, 2006, vol. II, European Mathematical Society, 2006, pp. 131-149.

[10] M.P.F. du Sautoy and A. Lubotzky, Functional equations and uniformity for local zeta functions of nilpotent groups, Amer. J. Math. 118 (1996), no. 1, 39-90.

[11] M.P.F. du Sautoy and D. Segal, Zeta functions of groups, New horizons in pro- $p$ groups, Progr. Math., Birkhæuser, Boston MA, 2000, pp. 249-286.

[12] M.P.F. du Sautoy and G. Taylor, The zeta function of $\mathfrak{s l}_{2}$ and resolution of singularities., Math. Proc. Cambridge Philos. Soc 132 (2002), no. 1, 57-73. 
[13] M.P.F. du Sautoy and L. Woodward, Zeta functions of groups and rings, Lecture Notes in Mathematics 1925, Springer Verlag, 2008.

[14] F.J. Grunewald, D. Segal, and G.C. Smith, Subgroups of finite index in nilpotent groups, Invent. Math. 93 (1988), 185-223.

[15] R.E. Howe, Kirillov theory for compact p-adic groups, Pacific J. Math. 73 (1977), no. 2, $365-381$.

[16] - On representations of discrete, finitely generated, torsion-free, nilpotent groups, Pacific J. Math. 73 (1977), no. 2, 281-305.

[17] E. Hrushovski and B. Martin, Zeta functions from definable equivalence relations, preprint, 2004.

[18] J.-I. Igusa, Universal p-adic zeta functions and their functional equations, Amer. J. Math. 111 (1989), 671-716.

[19] A. Jaikin-Zapirain, Zeta function of representations of compact p-adic analytic groups, J. Amer. Math. Soc. 19 (2006), no. 19, 91-118.

[20] E.I. Khukhro, p-automorphisms of finite p-groups, London Mathematical Society Lecture Note Series 146, Cambridge University Press, 1998.

[21] B. Klopsch, Zeta functions related to the pro-p group $S L_{1}\left(\Delta_{p}\right)$, Math. Proc. Cambridge Philos. Soc. 135 (2003), 45-57.

[22] B. Klopsch, C. Voll, Zeta functions of 3-dimensional p-adic Lie algebras, Oberwolfach Preprints OWP 2007-10.

[23] A. Lubotzky and A.R. Magid, Varieties of representations of finitely generated groups, Mem. Amer. Math. Soc. 58 (1985), no. 336, xi+117 pp.

[24] A. Lubotzky and D. Segal, Subgroup growth, Birkhäuser Verlag, 2003.

[25] C. Nunley and A.R. Magid, Simple representations of the integral Heisenberg group, Contemp. Math. 82 (1989), 89-96.

[26] D. Segal, Polycyclic groups, Cambridge Tracts in Mathematics 82, CUP, 1983.

[27] J.-P. Serre, Lie algebras and Lie groups, Second edition, Lecture Notes in Mathematics 1500, Springer Verlag, 1992.

[28] Richard P. Stanley, Enumerative combinatorics, Cambridge Studies in Advanced Mathematics, 49, vol. 1, Cambridge University Press, 1997.

[29] W. Veys and W.A. Zuniga-Galindo, Zeta functions for analytic mappings, logprincipalization of ideals, and Newton polyhedra, Trans. Amer. Math. Soc. 306 (2008), 2205-2227.

[30] C. Voll, Zeta functions of groups and enumeration in Bruhat-Tits buildings, Amer. J. Math. 126 (2004), 1005-1032.

[31] - Functional equations for local normal zeta functions of nilpotent groups, Geom. Func. Anal. (GAFA) 15 (2005), 274-295, with an appendix by A. Beauville.

[32] - Counting subgroups in a family of nilpotent semidirect products, Bull. London Math. Soc. 38 (2006), 743-752.

[33] J. Wlodarczyk, Simple Hironaka resolution in characteristic zero, J. Amer. Math. Soc. 18 (2005), no. 4, 779-822.

[34] L. Woodward, Zeta functions of groups: computer calculations and functional equations, Ph.D. thesis, University of Oxford, 2005.

\section{EMAil: C.Voll.98 at CANTAB.NET}

School of Mathematics, University of Southampton, Highfield, SO17 1BJ, UNITED KINGDOM. 